\title{
NISTIR 6300
}

The AUTONAV/DOT Project: Baseline Measurement System for Evaluation of Roadway Departure Warning System

\section{Sandor Szabo \\ Karl Murphy \\ Maris Juberts}

U.S. DEPARTMENT OF COMMERCE

Technology Administration

National Institute of Standards

and Technology

Intelligent Systems Division

Gaithersburg, MD 20899-8230 


\section{NISTIR 6300}

\section{The AUTONAV/DOT Project: Baseline Measurement System for Evaluation of Roadway Departure Warning System}

\section{Sandor Szabo \\ Karl Murphy \\ Marls Juberts}

U.S. DEPARTMENT OF COMMERCE

Technology Administration

National Institute of Standards

and Technology

Intelligent Systems Division

Gaithersburg, MD 20899-8230

March 1999

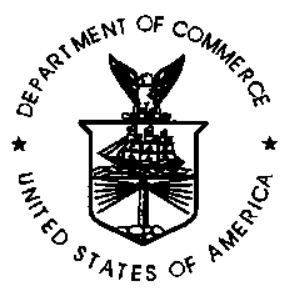

U.S. DEPARTMENT OF COMMERCE William M. Daley, Secretary

TECHNOLOGY ADMINISTRATION

Gary R. Bachula, Acting Under Secretary for Technology

NATIONAL INSTITUTE OF STANDARDS

AND TECHNOLOGY

Raymond G. Kammer, Director 


\title{
The AUTONAV / DOT Project: Baseline Measurement System For Evaluation of Roadway Departure Warning Systems
}

\author{
Submitted to \\ U.S. Department of Transportation \\ National Highway Traffic Safety Administration \\ Office of Collision Avoidance Research \\ Washington, D.C. 20590
}

\author{
Submitted by \\ Sandor Szabo, Karl Murphy and Maris Juberts \\ National Institute of Standards and Technology \\ Intelligent Systems Division \\ Building 220, Rm. B127 \\ Gaithersburg, MD 20899 \\ Army Research Laboratory \\ Aberdeen Proving Grounds \\ Aberdeen, MD
}

January 27, 1999 



\section{$\underline{\text { Table of Contents }}$}

EXECUTIVE SUMMARY ..................................................................................................................... 1

1 INTRODUCTION

2 SCENARIO FOR A ROADWAY DEPARTURE SYSTEM EVALUATION ............... 4

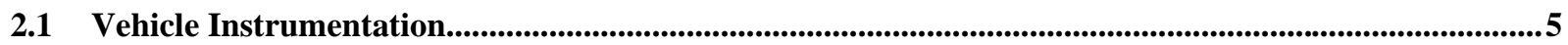

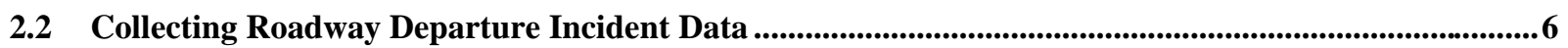

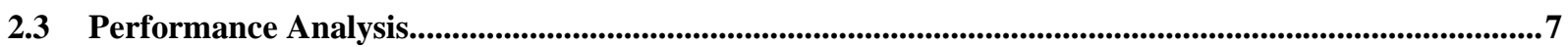

3 BASELINE MEASUREMENT SYSTEM....................................................................... 10

3.1 Inertial Navigation System..............................................................................................................................

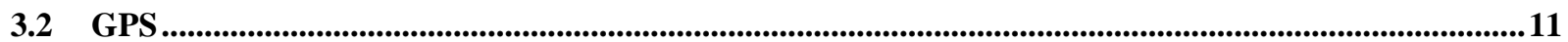

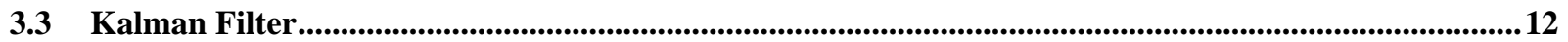

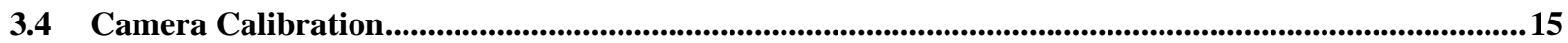

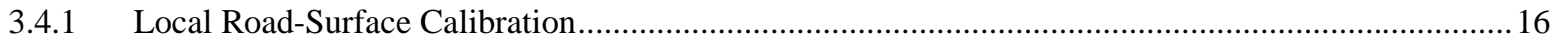

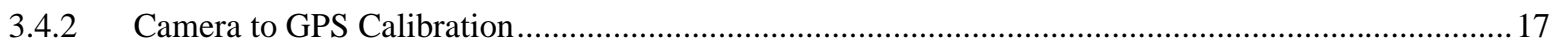

3.5 Video Digital Recording System .............................................................................................................................22

4 EVALUATION OF BASELINE MEASUREMENT SYSTEM ................................ 25

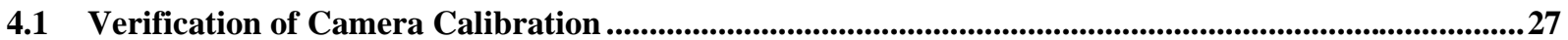

4.2 Static Measurements ..................................................................................................................................................28

4.3 Dynamic Measurements and Latency Calculation ...............................................................................................30

4.4 Affects of GPS Dropout.................................................................................................................................32

4.5 Lane Position Measurements........................................................................................................................................38

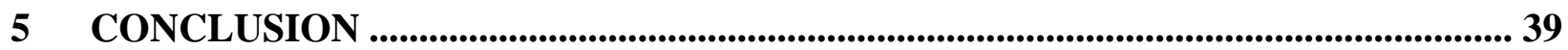

APPENDIX A: AUTONAV PROGRAM DESCRIPTION.......................................................... 40

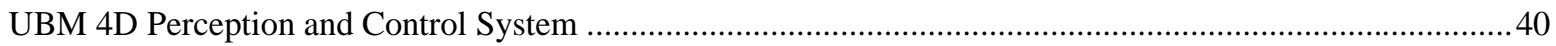

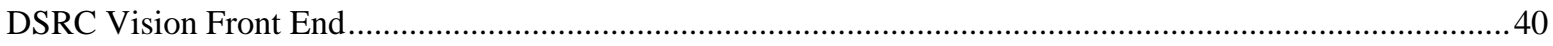

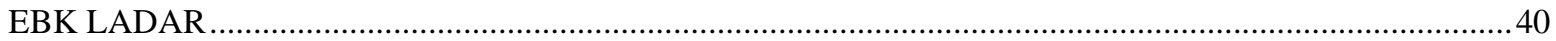

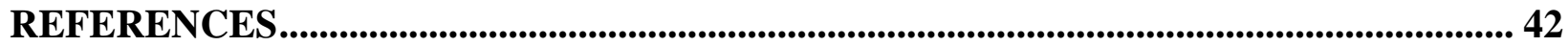




\section{List of Tables}

Table 1. The possible outcomes of a warning system evaluation at each instance in time.................................. 8

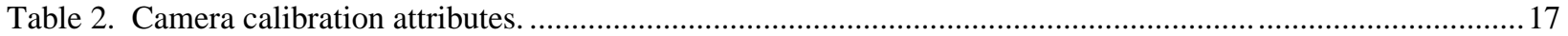

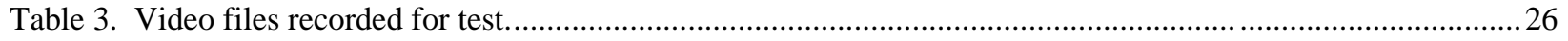

Table 4. Navigation data files corresponding to video files in Table 3 .........................................................26

Table 5. Values of visually acquired nails and their distance from the origin (i.e., the known survey location of the

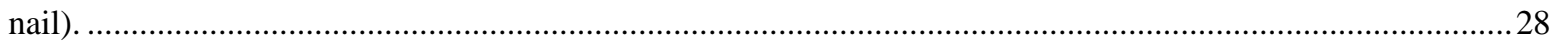

Table 6. Visual-based measurements of nail (see Figure 18) with distance from manual surveyed coordinates of the

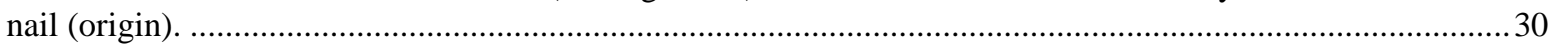

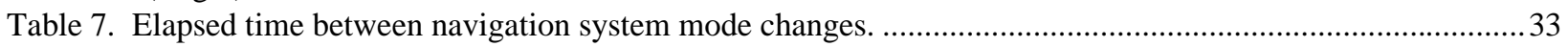




\section{List of Figures}

Figure 1. Vehicle instrumentation for evaluating performance of a roadway departure warning system. ..................5

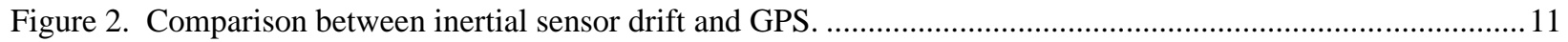

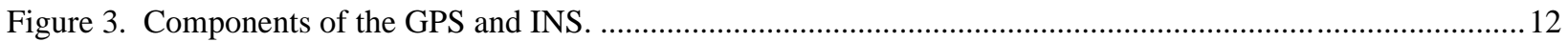

Figure 4. A complementary filter is used to estimate the true position of the vehicle. ......................................... 13

Figure 5. The Kalman filter computes estimated drift and noise in the Northing direction. ................................... 14

Figure 6. Picture of downward pointing camera mounted on side of vehicle. .................................................... 15

Figure 7. Calibration target is used to define a local road-surface origin $(x, y)$ at top left control point....................16

Figure 8. Vehicle coordinate systems describing camera-to-GPS calibration..................................................... 18

Figure 9. Derivation of position transformation between the local road surface and the GPS coordinate systems..... 19

Figure 10. Two points are required to derive the rotation transformation between the local road surface and the GPS

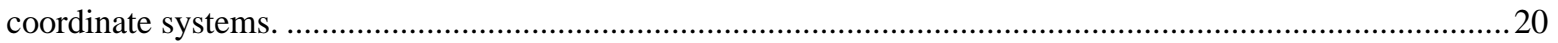

Figure 11. Derivation of rotation transformation between camera and GPS coordinate systems............................21

Figure 12. Components of the Video Digital Recording System. ..............................................................23

Figure 13. Picture of the BMS Video/Data Digital Recording System...................................................23

Figure 14. A single frame recorded by BMS showing two survey nails highlighted using black and white paint......26

Figure 15. Vehicle driven along a half figure-eight trajectory along with survey nails acquired using video at

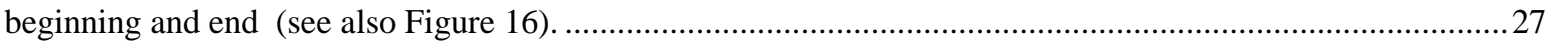

Figure 16. Filtered and unfiltered (raw) GPS coordinates of nail at beginning and end of half figure 8 trajectory....28

Figure 17. Trajectory of vehicle during test to acquire nail at beginning and end of run...................................29

Figure 18. Results of visually acquiring survey nail at the beginning and end of several runs. ................................3 30

Figure 19. The error due to latency is shown in the discrepancy between the coordinates of the surveyed nail (surveyedNail) and the visually acquired nail (videoNail) as the vehicle (filtered_gps) drives by at $10.75 \mathrm{~m} / \mathrm{s} .31$

Figure 20. A run out the NIST gate onto Muddy Branch Rd and then back to the start showing mode changes in the navigation system $(\mathrm{DR}=$ DeadReckoning, DGPS $=$ Differential GPS, cpDGPS = carrier phase DGPS $) \ldots \ldots \ldots . . . .32$

Figure 21. Vehicle position updates during section of trajectory labeled "a" in Figure 20...................................34

Figure 22. Vehicle position updates during section of trajectory labeled " $b$ " in Figure 20..................................35

Figure 23. Vehicle position updates during section of trajectory labeled "c" in Figure 20..................................36

Figure 24. Nail locations, derived from navigation system (filtered_gps) and raw GPS (unfiltered_gps) data, at the beginning and end of run in Figure 20.

Figure 25. Trajectory of vehicle and location of outside lane marker shown with minimum and maximum distance between vehicle and lane labeled ( $\mathrm{min} / \mathrm{max}$ : distance from lane in meters, GPS record number)....................38 


\section{$\underline{\text { Executive Summary }}^{\mathrm{i}}$}

This report describes a set of performance metrics and a performance measurement system developed for the evaluation of roadway departure warning systems. The work is part of an effort by the Department of Transportation's National Highway Traffic and Safety Administration (NHTSA) to develop quantitative measures of performance for the evaluation of highway safety systems. The work is also part of an effort to promote dual-use technologies between the Department of Transportation and the Department of Defense. The Department of Commerce's National Institute of Standards and Technology undertook the development of the performance measurement system.

The approach for quantitative evaluation rests on establishing a baseline measurement of roadway departure that is of higher accuracy than the warning system under test. The output of the warning system is then compared to the baseline measurement to produce a quantitative measure of performance. The baseline measurement system is installed on a vehicle with the warning system. The baseline system achieves high accuracy by aiming a calibrated camera onto an area of the road adjacent to the vehicle. As the vehicle is driven and roadway departure maneuvers are performed, the output of the video camera, the vehicle's position (from a global positioning system and inertial sensors), and the output of the warning system are synchronized and digitally recorded. After a vehicle run is complete, a suite of software is used to automatically analyze the baseline video data to determine roadway departure. Since the video and other data remain available after the vehicle run, an operator (to establish the credibility of the baseline measurement) can verify a roadway departure. Also, the data can be analyzed to provide insight into the cause of a warning system failure (e.g., insufficient roadway markings, high lateral velocity, etc.). The time of departure is compared with the warning system output to determine the reliability, failure and false alarm rates of the warning system.

This report describes the performance metrics and the baseline measurement system. It also describes efforts to determine the accuracy of the baseline system in determining the time and position of roadway departures. In addition, the precise, synchronized recording of video and GPS data proved useful in determining the accuracy and the delays of GPS when measuring the position of a moving vehicle. The baseline measurement system is now sufficiently mature to be used to evaluate roadway departure warning systems. The measurement system also serves as a promising platform to perform a wide range of other evaluations.

\footnotetext{
i This paper was prepared by U.S. Government employees and is not subject to copyright. The use of commercial equipment or products is for demonstration only and does not imply recommendation or endorsement by NIST.
} 


\section{Introduction}

One of the goals of the Department of Transportation, National Highway Traffic and Safety Administration (NHTSA) is to improve safety on U.S. highways. An avenue for improved safety is the use of driver warning systems for collision avoidance and roadway departure. Warning systems for roadway departure rely on advanced sensors and processing algorithms to warn the driver when the vehicle is leaving the road unintentionally. The U.S. Department of Defense, under the AUTONAV (Autonomous Vehicle Navigation Control System) project, is looking at similar sensors and algorithms for controlling autonomous ground vehicles. The basis for the interaction between DOT and DOD is to promote dual-use technologies for the improvement of surface transportation safety. The Army Research Lab (ARL) has provided the National Institute of Standards and Technology (NIST), the primary research participant, with a vehicle and various sensing and control components (see Appendix A: Autonav Program Description) to serve as the test-bed to evaluate warning systems and to develop methods for performance measurement. This report describes a set of performance metrics and a performance measurement system developed for the evaluation of roadway departure warning systems.

A primary function of a roadway departure warning system is to detect the location of the roadway boundaries. The location of the roadway must be detected sufficiently forward of the vehicle to provide time for both the warning system to determine whether the vehicle is on a course to leave the lane, and for the driver to take the appropriate action. The warning system must operate on a wide variety of roads, day or night, and under a wide range of weather conditions. The main effort in the AUTONAV/DOT project was to develop a method for quantitatively measuring the performance of the warning system under these varied conditions. The primary measure of performance is the reliability of the warning system to detect roadway departure incidents and to reject false alarms. The method uses a baseline measurement system (BMS) to obtain a baseline of how a warning system is expected to perform. The baseline is compared to the warning system's actual performance in order to determine a measure of reliability. Section 2 presents a scenario of how a warning system is evaluated using the BMS. The components of the BMS are described in section 3. Several tests were conducted to validate the measurement capabilities of the BMS. The results of the BMS tests are presented in section 4.

The BMS was developed based on NIST's experience ${ }^{1}$ with systems that have demonstrated the capability to perform lane tracking and that could serve as a potential warning system. During the time of the work, there were no commercially available systems, but recent announcements have been made that products will soon be available. The final method for evaluation of these warning systems will depend on the goals of the manufacturer and of the evaluators. For example, the evaluation may also seek to obtain the response of the driver to the warning system's operation. The intent of the efforts described in this report is to build an understanding of the types of measurements that can be performed and to demonstrate a method of evaluation. More work needs to be done to refine the measures of performance, to increase the number of measurement capabilities, and to improve the accuracy of the measurements. The authors 
express gratitude to Alhad Chande and August Burgett of the National Highway Traffic Safety Administration's Office of Crash Avoidance Research for their insights, perseverance and contributions toward the completion of this project and the report 


\section{Scenario for a Roadway Departure System Evaluation}

Several types of evaluations can be conducted to obtain an overall performance evaluation of a roadway departure warning system. A preliminary evaluation is usually conducted prior to a field operational test in order to determine the safety and reliability of the warning system under a controlled set of circumstances. The results of a preliminary evaluation are used to design and implement a full-scale field operational test involving a large number of drivers operating under normal everyday driving conditions. A scenario for a preliminary evaluation of a roadway departure warning system is described in this section. This scenario drove the design and implementation of a performance measurement system called the baseline measurement system (BMS). The following list highlights some general attributes of a preliminary evaluation.

1. Ability to generate a large number of roadway departure incidents.

2. Ability to record the response of the warning system to each incident.

3. Ability to establish a baseline, that is, the correct operation expected from the warning system, and to compare the output of the warning system against the baseline in a quantitative manner.

4. Ability to perform evaluations on real roads - not just on a closed test tracks.

5. Ability to perform evaluations in wide variety of environmental conditions: lighting, weather, etc.

6. Ability to perform the evaluations in a cost-effective manner.

The first step of an evaluation is to instrument the vehicle with the warning system and the BMS. An overview of the vehicle instrumentation is given in section 2.1. Once the instrumentation is in place and calibrated, the remaining procedures in an evaluation plan consist of a series of testdrives and data collections described in section 2.2. Section 2.3 describes how the data is analyzed to produce various measures of performance that describes the reliability of the warning system. 


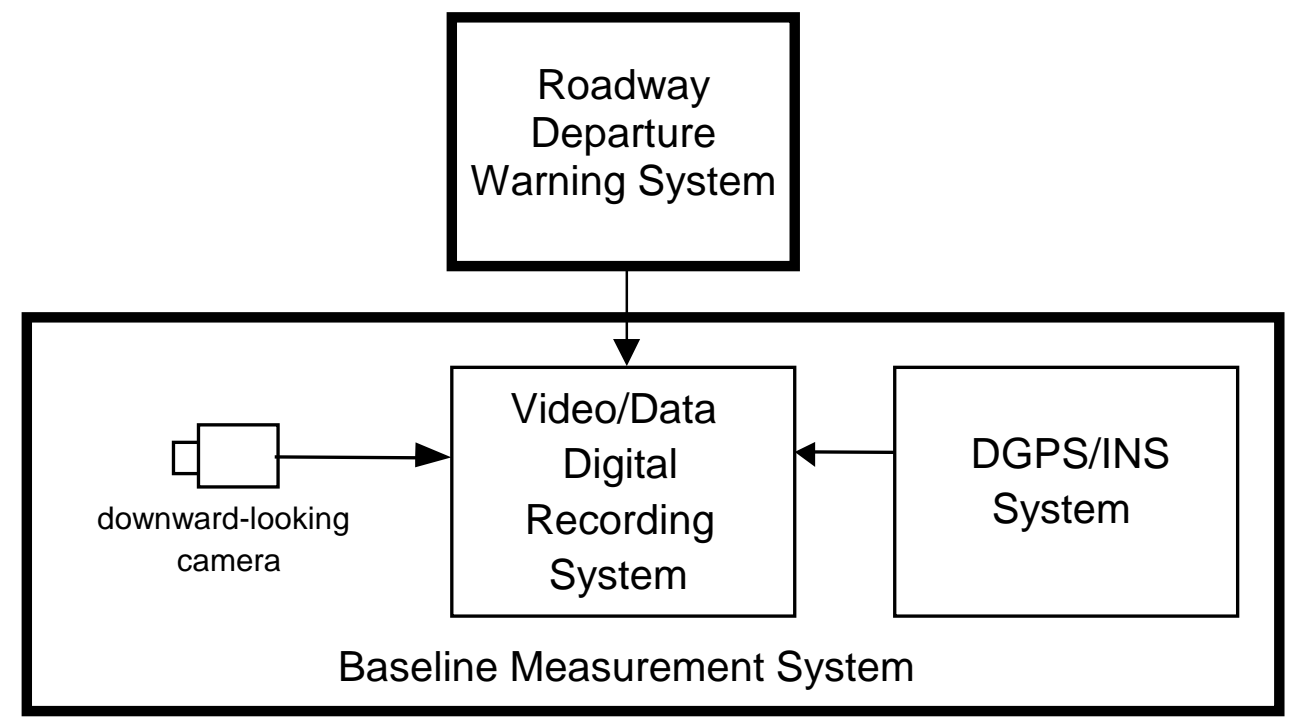

Figure 1. Vehicle instrumentation for evaluating performance of a roadway departure warning system.

\subsection{Vehicle Instrumentation}

The first step to a preliminary evaluation of a roadway departure warning system is to install the warning system and the baseline measurement system on the vehicle. For scenario purposes, it is assumed that the warning system is vision-based, and the manufacturer installs the system on the vehicle and performs any required calibrations. Figure 1 shows the components of the BMS that are installed on the vehicle. The Video/Digital Recording subsystem consists of a calibrated camera and a digital recorder. The camera is used to measure the position of the vehicle relative to a lane marker. It is mounted on the vehicle and points to the side and downward as close to perpendicular as possible. Currently, only a single camera is used to capture either the inside or outside lane marker. There are several methods to extend the BMS to multiple cameras so that both markers can be detected. The camera is calibrated so that image coordinates in pixels can be transformed into road-surface coordinates in millimeters.

The GPS/INS subsystem measures vehicle position and velocity using a carrier phase differential GPS augmented with an inertial navigation system. When GPS/INS data is available, the camera can be calibrated further so that image coordinates can be transformed into GPS coordinates (northing and easting). It should be noted that a warning system can be evaluated without the GPS/INS subsystem. The camera system data provides the distance to the lane marker, which is sufficient for determining when a vehicle departs a lane. The GPS/INS subsystem is necessary when the evaluation also requires where the vehicle departs the lane. Both video and navigation data are stored digitally on an array of hard disks. Digital storage on disk enables synchronization of all the data. In addition, the data can be examined immediately to ensure its quality. Finally, the analysis of the data is not limited by the onboard processor's requirement to respond to real-time events. 
The third component shown in Figure 1 is the warning system under evaluation. An interface is developed between the BMS and the warning system so that roadway departure warnings generated by the system can be recorded along with the BMS video and GPS/INS data. Other types of warning system data may be recorded, including:

1. Loss of lane marker tracking. This may result when the lane markers fade or disappear.

2. Time-to-departure from lane. The estimated time until the vehicle crosses the lane marker.

3. Distance from lane marker and closing rate to lane marker.

4. Lane curvature.

\subsection{Collecting Roadway Departure Incident Data}

The purpose of the data collection is to obtain data describing the performance of the warning system under a wide variety of operational conditions. The conditions are selected to closely mimic real driving environments. The data provides insight into the accuracy of the system in detecting a roadway departure and in rejecting false alarms. Generating incidents for a rear-end collision avoidance system is difficult, but this is not the case for a roadway departure warming system. Driving the vehicle over various roads and intentionally crossing over the lane markers is sufficient for a preliminary evaluation.

The operational parameters for data collection are the various conditions that affect the performance of the warning system. Prior to the evaluation, each parameter is identified and the space the parameter spans is defined. For example, lighting is one parameter and it spans from full daylight to nighttime. When possible, the parameter is measured in order to quantify under what conditions the data was collected. The following is a brief list of some parameters that may affect system performance.

1. Lane proximity - distance between the vehicle and lane marker.

2. Lane departure rate - rate at which vehicle can approach lane marker.

3. Lane departure frequency - amount of time between lane crossings.

4. Road surface types - asphalt, concrete, etc.

5. Road surface quality - cracks, potholes, etc.

6. Lane marker contrast - bright, faded, etc.

7. Lane marker type - solid, stripped, etc.

8. Lane types - merge, split, intersection, etc.

9. Lane curvature - curvature of road the system can monitor.

10. Light intensity - direct sunlight, clouds, dusk, night, etc.

11. Weather condition. Dry, rain, snow, etc.

Once the BMS is installed on the vehicle and calibrated, the process of collecting data is straightforward. First, a time and place is selected based on the type of data needed, i.e., nighttime, rainy weather on interstate. The driver turns on the BMS and starts driving. The driver performs maneuvers that test the response of the warning system to both lane crossings and non-lane crossings. The data is collected in relatively short segments of 30 to 60 minutes each. During each data collection, the driving is divided into two phases. In the first phase, no lane crossings are performed in order to gather data characterizing the false alarm rate of the 
warning system. In the second phase, a variety of maneuvers are performed that cause the vehicle to cross a lane marker. This can be done in a safe manner by performing the maneuver on sections of road where no other vehicles are close by. Also, run-off-road maneuvers can be performed along roads where sufficient asphalt surface exists on the other side of the lane marker to permit safe control of the vehicle after the lane marker is crossed.

The steps performed by the driver to collect data are summarized in the following list.

1. Select time and place for data collection in order to satisfy desired operational parameters.

2. Turn on BMS recording and verify data quality.

3. Drive down center of lane for 10 minutes.

4. Decrease distance to lane marker in increments of $15 \mathrm{~cm}$ (6 inches) and maintain distance for 1 minute. Stop decreasing distance prior to contact with lane marker. Return to center and perform same steps toward opposite lane marker (assumes BMS records lane position on both sides). Return to lane center when done.

5. Slowly oscillate position of vehicle within lane without touching lane marker. In safe manner, slowly increase frequency of oscillation.

6. Select section of road where lane crossings can be performed.

7. Perform several lane changes in both directions.

8. Slowly oscillate position of vehicle so as to cross lane marker repeatedly. In safe manner, slowly increase frequency of oscillation.

9. Turn off BMS recording and verify data quality.

10. Make entries describing conditions during data collection not automatically recorded by the BMS (weather, road quality, etc.).

After the collection run is complete, the data is transferred from the vehicle to a workstation where warning system performance is evaluated using BMS analysis software.

\subsection{Performance Analysis}

The performance of the warning system is judged by its reliability, that is, the ability to warn when a lane departure takes place and to reject false alarms. A quantitative measure of performance (MOP) for reliability of the warning system is defined as:

$$
\text { general reliability }=\left(\frac{\text { successful outcomes }}{\text { all possible outcomes }}\right) \cdot 100=\left(\frac{T P+T N}{T P+T N+F P+F N}\right) \cdot 100
$$

The general reliability of the warning system is obtained by comparing the output of the warning system against the data collected by the BMS. In each video frame, both the BMS and the warning system measure the distance between the vehicle and the lane marker. For a basic system that warns when the lane edge is crossed, if the distance goes to zero, then the vehicle has departed the road and the warning system should signal a roadway departure incident. Column 1 in Table 1 labels the possible responses of the warning system. The remaining entries in the table describe the possible outcomes of a comparison between the warning system and the BMS data. A true positive (TP) outcome indicates that both the warning system and the BMS data indicate a roadway departure incident. A false positive (FP) outcome, or a false alarm, occurs when the 
warning system issues a warning and none is detected in the BMS data. A false negative outcome, potentially the most catastrophic result, occurs when the warning system completely misses a roadway departure. Finally, a true negative indicates that the warning system is not responding and none exists.

\begin{tabular}{|c|c|c|}
\hline Warning System Response & $\begin{array}{c}\text { BMS indicates roadway } \\
\text { departure }\end{array}$ & $\begin{array}{c}\text { BMS indicates no roadway } \\
\text { departure }\end{array}$ \\
\hline $\begin{array}{c}\text { warning system warns of } \\
\text { roadway departure }\end{array}$ & $\begin{array}{c}\text { True Positive (TP) } \\
\text { Correctly warns of roadway } \\
\text { departure }\end{array}$ & $\begin{array}{c}\text { False Positive (FP) } \\
\text { False alarm }\end{array}$ \\
\hline $\begin{array}{c}\text { warning system does not warn } \\
\text { of roadway departure }\end{array}$ & $\begin{array}{c}\text { False Negative (FN) } \\
\text { Misses roadway departure }\end{array}$ & $\begin{array}{c}\text { True Negative (TN) } \\
\text { Correctly does not warn of } \\
\text { roadway departure. }\end{array}$ \\
\hline
\end{tabular}

Table 1. The possible outcomes of a warning system evaluation at each instance in time.

The general reliability metric rewards a warning system for correctly not warning of a roadway departure (true negative). This leads to a problem with the metric because the majority of the data will produce true negative outcomes. This bias may produce a high reliability value even when the warning system misses all roadway departures. For example, consider the following case where the warning system misses all 10 lane departures $(\mathrm{TP}+\mathrm{FN}$ defines the total number of lane departures) and yet scores a general reliability of $99 \%$ :

$$
\begin{aligned}
& T P=0 \\
& T N=1000 \\
& F P=0 \\
& F N=10 \\
& \text { general reliability }=\left(\frac{0+1000}{0+1000+0+10}\right) \cdot 100=99 \%
\end{aligned}
$$

An alternative metric focuses on the reliability of the warning system in critical situations when the vehicle departs the road.

$$
\text { critical reliability }=\left(\frac{T P}{T P+F N}\right) \cdot 100
$$

A critical reliability of $100 \%$ indicates that the warning system never failed to detect a roadway departure when one occurred. Based on the example above, the critical reliability would be $0 \%$ and not $99 \%$. The failure rate of the warning system can be derived from the warning system's critical reliability.

$$
\text { failure rate }=1-\text { critical reliability }=\left(\frac{F N}{T P+F N}\right) \cdot 100
$$


The false alarm rate of the warning system is the number of false positives over some number of possible outcomes. The question is over what range of possible outcomes is the rate calculated?

$$
\text { false alarm rate }=\left(\frac{F P}{T P+T N+F P+F N}\right) \cdot 100
$$

Again, including a large number of true negative outcomes in the denominator will bias the false alarm rate toward a smaller value. Since the false alarm rate is more of an annoyance than a failure, and false alarms can occur at any time (not just during critical moments), the rate is defined over all possible outcomes.

A report is generated that summarizes the results of the analysis for all sets of data. The report specifies the state of the operational parameters listed in section 2.2 and the metrics described above. The metrics will vary for each set of data. For example, the reliability may be $98 \%$ during daylight hours and $88 \%$ at night. A cumulative reliability is computed based on the results under all the conditions.

The preceding scenario provides one example of how the BMS is used to evaluate a roadway departure warning system. Reliability is the primary measure of performance but additional measures may be appropriate. These measures will be developed as the goals of the evaluation of a specific warning system are finalized. The BMS is a general-purpose system and can be programmed to meet a wide range of performance analysis requirements. The remaining sections in this report provide details of the design, integration, and capability of the BMS. 


\section{Baseline Measurement System}

The BMS is primarily a data collection system designed to capture video, GPS data, and the output from a countermeasure system under evaluation. A digital recording system is used to capture and synchronize all the data more easily. Recording the data, as opposed to performing the evaluation in real-time, has several advantages. First, and foremost, is the ability to analyze the data in order to reduce the uncertainty of the reference. Without the ability to analyze the data, it is difficult to make the assertion that the BMS serves as the reference for other measurements. As an example, suppose a roadway departure system under evaluation reports that the vehicle crossed a lane marker at a particular position or instance in time. The BMS, on the other hand, reports that the vehicle did not cross a lane marker at that point. Which system is correct? Recording the entire event enables the evaluator to examine the data and judge which is more accurate. With the visual data recorded, additional methods can be used to improve the uncertainty. For example, survey markers can be used as a reference to improve position measurements. Another advantage of recording the data is the ability to conduct a variety of analyses that may not be possible under real-time constraints.

The BMS consists of two distinct subsystems. The GPS/INS subsystem is responsible for measuring vehicle position in GPS coordinates. The next section covers the Inertial Navigation System and the Differential GPS separately. The focus of the descriptions is to present the operating characteristics and the accuracy of each component as if they were used individually. The section on the Kalman filter describes how the INS and GPS measurements are combined together to achieve a measurement system that exhibits the best characteristics of each.

The second subsystem of the BMS is the video/data digital recording system (VDDRS). The VDDRS is based on a ruggedized, off-the-shelf, Silicon Graphics Inc. O2 computer system. The $\mathrm{O} 2$ records camera input and data from the GPS/INS subsystem onto an array of disks. The camera is calibrated so that points in the image can be transformed into GPS coordinates. A variety of analysis software exists that automatically locates a lane marker in a video frame, detects lane crossings, and plots the results of an evaluation. The camera calibration and the digital recorder are covered in the remaining portions of this section.

\subsection{Inertial Navigation System}

The U.S. Army's Modular Azimuth and Positioning System, MAPS ${ }^{2}$, is an inertial navigation system containing three ring laser gyros, three accelerometers, and a rear axle odometer. MAPS can supply orientation and translation data at $5 \mathrm{~Hz}$. A Navigation Interface Unit (NIU), previously developed by Alliant Tech, is used to increase the update rate. The NIU requests orientation data from the MAPS, which is available by itself at $25 \mathrm{~Hz}$, and taps into the odometer to measure the distance traveled. It combines the odometer and heading data to generate vehicle position data at $25 \mathrm{~Hz}$.

The MAPS/NIU position measurement drifts as the vehicle moves, due mainly to wheel slippage. Our estimate of the drift rate of the system on pavement and in dry grass is slightly more than $0.1 \%$ of the distance traveled. The estimate was obtained by first marking on the pavement the 
initial location of the tires and recording the vehicle position reported by the system. The vehicle was driven along a course and returned as close as possible to the initial tire locations. The output of the system was then compared to the initial vehicle position and the difference was calculated to be $0.1 \%$ of the distance traveled. The runs were not repeated enough to obtain a useful standard deviation of the estimate. Because the system uses odometry obtained from the wheels, the system drifts more when driving on slippery surfaces and steep slopes.

Figure 2 shows the inertial dead reckoning system drifting as the vehicle is driven around a square track. The inertial system drifts to the north and to the west by a similar amount each pass. In this example, the vehicle was driven through patches of snow causing the inertial dead reckoning system to drift at $0.4 \%$ of distance traveled.

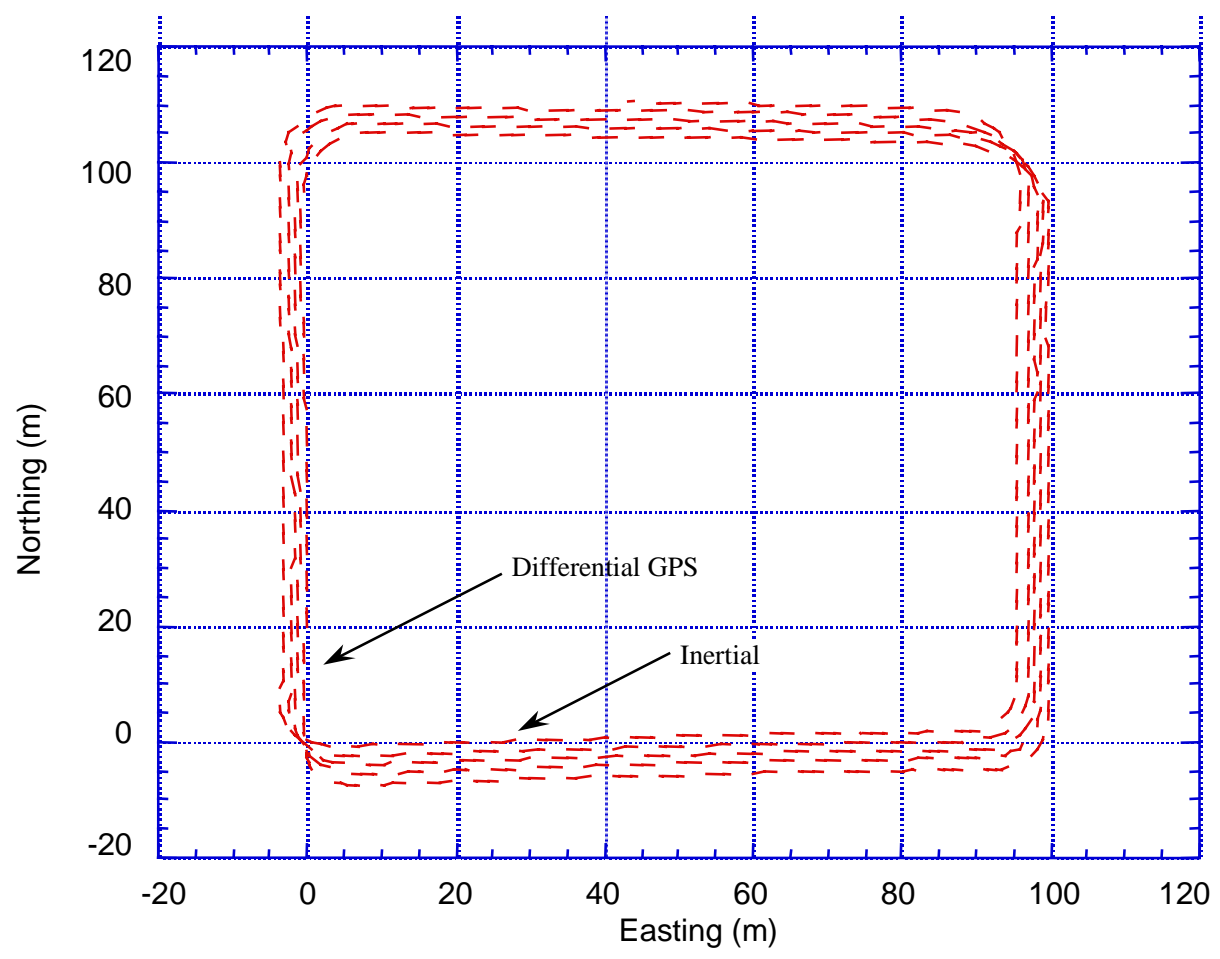

Figure 2. Comparison between inertial sensor drift and GPS.

\subsection{GPS}

The satellite based Global Positioning System, GPS, has a stated accuracy ${ }^{\text {ii }}$ of $100 \mathrm{~m}$ in the standalone mode for civilian receivers and around $10 \mathrm{~m}$ to $30 \mathrm{~m}$ for stand alone military receivers. To improve accuracy, differential GPS is used. Figure 3 shows the components of the differential GPS for the NIST vehicle. A stationary receiver at a known location, in this case, located on top of NIST Administration Building, transmits perceived position errors over a data radio to the

\footnotetext{
ii These GPS accuracy figures are from the 1994 Federal Radionavigation Plan. The figures express the value of two standard deviations of radial error from the actual antenna position to an ensemble of position estimates made under specified satellite conditions.
} 
moving GPS receiver onboard the vehicle. The vehicle receiver uses this data to improve its position estimate. Using this technique, accuracies from several meters down to sub-meter can be obtained. Even higher accuracy can be obtained by including carrier phase measurements in the transmissions. Using long-term averaging and post processing, carrier phase differential GPS can achieve accuracies of a centimeter or better while the receiver is stationary. An important feature of carrier phase differential GPS, available within the last two years on commercial systems, is the ability to resolve the cycle integer ambiguities of the carrier phase while the remote receiver is moving. This allows the vehicle to lose satellite lock (caused by driving under a bridge, near buildings, through foliage, etc.) and then reacquire phase lock on the fly. When there are not enough satellites visible for GPS to calculate an accurate position, the GPS/INS system relies solely on the inertial dead reckoning of the MAPS and NIU.

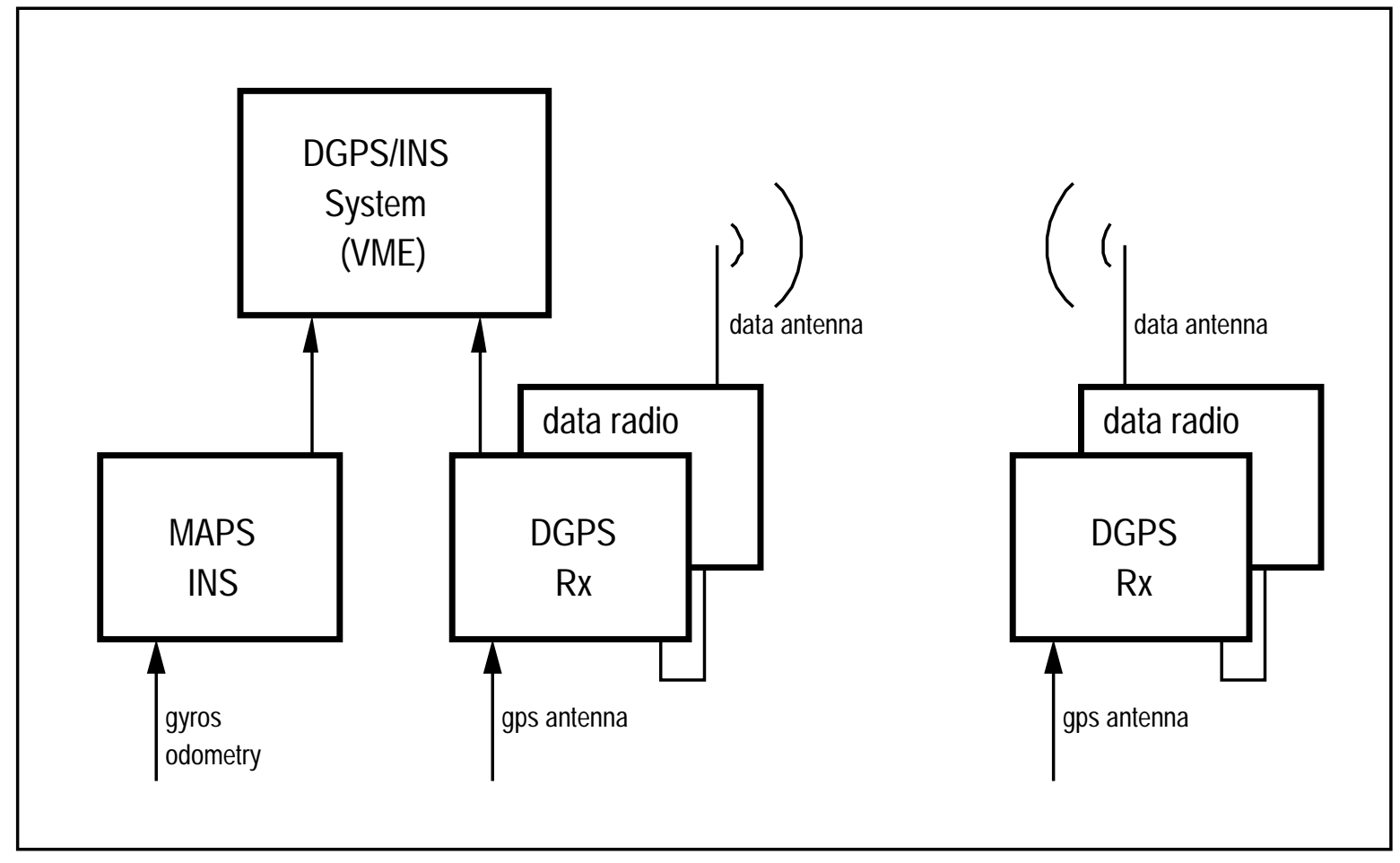

Figure 3. Components of the GPS and INS.

\subsection{Kalman Filter}

Both the INS and the GPS systems have advantages and disadvantages. INS has a higher data rate of $25 \mathrm{~Hz}$, but it drifts. The GPS system does not drift significantly over time, but it has a noise component, a slower data rate of $2 \mathrm{~Hz}$, and is not always available. To get the best of both systems, their outputs are combined using a complementary filter, shown in Figure 4. 


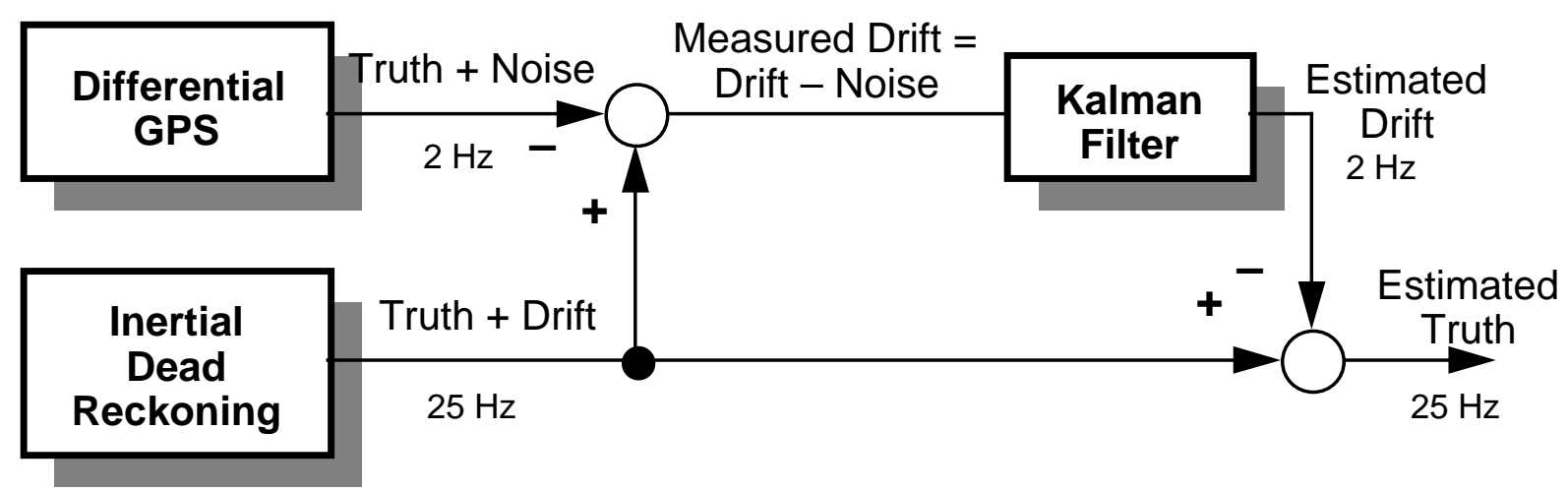

Figure 4. A complementary filter is used to estimate the true position of the vehicle.

The vehicle is at some position we will call truth. Both navigation sensors try to measure this but have some inherent error. The inertial dead reckoning system reports truth with an error we will call drift, and the GPS also reports truth but with an error we will call GPS noise. Taking the difference between the output of the two sensors cancels the true position and results in the difference between the drift and the GPS noise. The Kalman filter tries to remove the noise and estimate the drift. Subtracting this drift estimate from the inertial dead reckoning system output yields an estimate of the true vehicle position. The Kalman filter runs once for each GPS update, producing drift estimates at $2 \mathrm{~Hz}$. However, truth is estimated at $25 \mathrm{~Hz}$ using the current inertial dead reckoning data and latest drift estimate. In general, drift changes slowly, allowing the calculation of position between GPS updates and during short GPS dropouts.

The Kalman filter uses a simple model for drift that assumes the drifts in Northing and in Easting are independent of each other. This results in two separate scalar filters. Recall that the filter is estimating drift and not position. The state and output model is

$$
\begin{aligned}
& d_{n+1}=d_{n}+w_{n} \\
& z_{n}=d_{n}+v_{n}
\end{aligned}
$$

where

$d_{n}$ is the drift at time $n$,

$w_{n}$ is the system noise with covariance $q_{n}$,

$z_{n}$ is the measured drift $\left(z_{n}=\right.$ Inertial $\left.-G P S\right)$, and

$v_{n}$ is the measurement noise with covariance $r_{n}$.

The system noise $w_{n}$ models wheel slippage and other factors that cause the drift to change. As drift tends to increase with the distance traveled, the covariance of the system noise, $q_{n}$, is modeled as being proportional to the distance traveled since the last GPS update. The measurement noise $v_{n}$ is the GPS noise. Its covariance, $r_{n}$, is modeled as a constant. 
The Kalman filter reduces to the following scalar equations (one set for Northing and an identical set for Easting)

$$
\begin{aligned}
& K_{n}=p_{n} /\left(p_{n}+r_{n}\right) \\
& \hat{d}_{n+1}=\hat{d}_{n}+K_{n}\left(z_{n}-\hat{d}_{n}\right) \\
& p_{n+1}=p_{n}\left(1-K_{n}\right)+q_{n}
\end{aligned}
$$

where

$K_{n}$ is the Kalman filter gain, and

$\hat{d}_{n}$ is the estimated drift with an error covariance $p_{n}$.

The recursive filter is started with an initial guess for $\hat{d}_{o}$ and $p_{o}$.

The position of the vehicle is then estimated as

$$
\sim \text { Truth }_{n}=\text { Inertial }_{n}-\hat{d}_{n}
$$

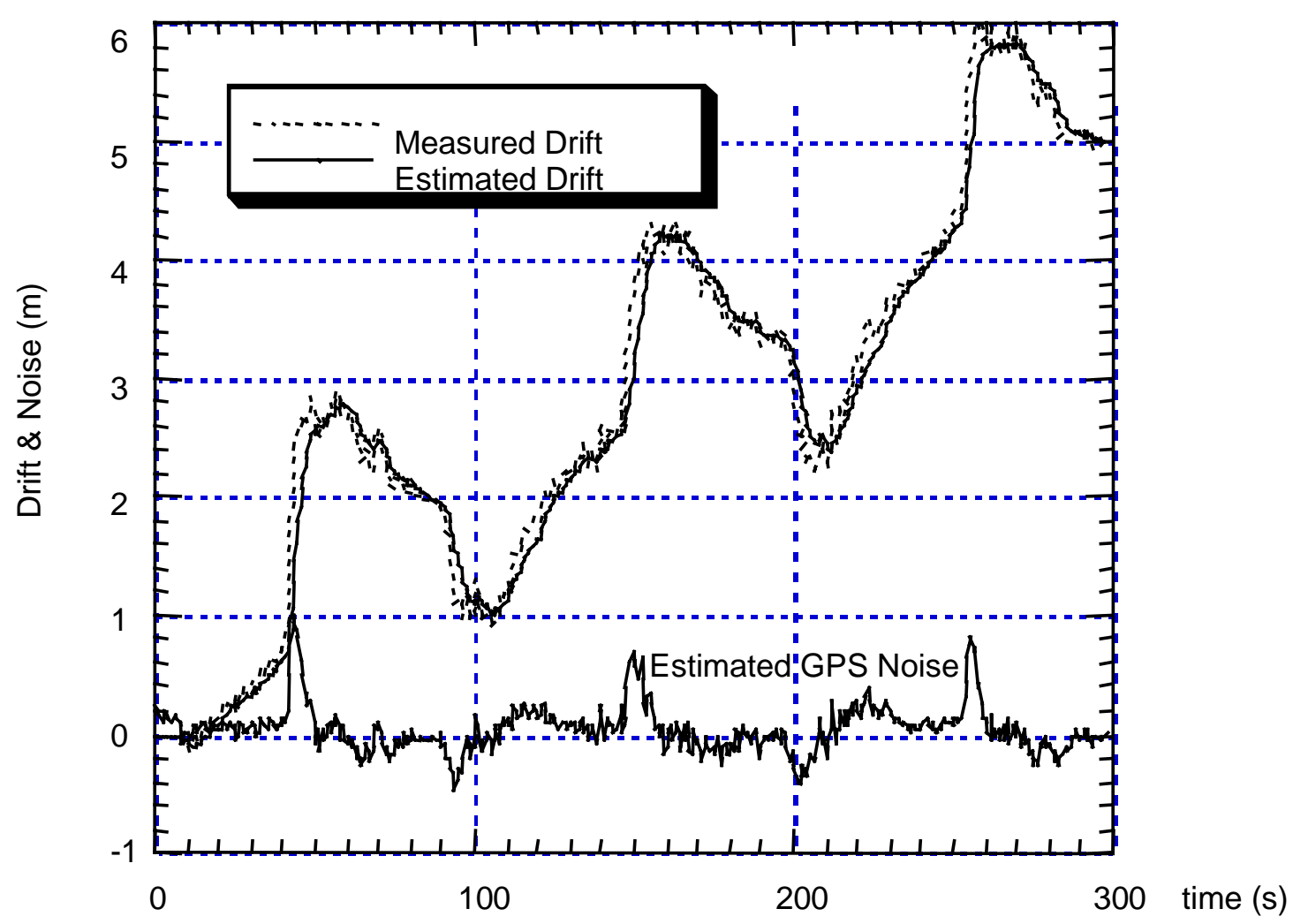

Figure 5. The Kalman filter computes estimated drift and noise in the Northing direction.

Figure 5 show the results of the Kalman filter for the first three laps of the square course shown in Figure 2. The GPS receiver is operating in the standard (non-carrier phase) differential mode, 
which provides position data to 1 meter (as claimed by the manufacturer). Both the measured drift, $z$, and the estimated drift, $\hat{d}$, increase in magnitude with time. During each lap the vehicle drifts in a similar direction at the same location producing a repetitive pattern about every $100 \mathrm{~s}$. The sensor noise, i.e., the GPS noise, cannot be determined exactly, but it can be estimated as ( $\mathrm{z}$ $-\hat{d})$. Note the peaks at $50 \mathrm{~s}, 150 \mathrm{~s}$, and $250 \mathrm{~s}$. These were caused when the vehicle slipped on a snow patch causing a large change in drift that the filter erroneously attributed to GPS noise. This mistake is corrected in a few seconds as further GPS updates corroborate the large change in drift.

\subsection{Camera Calibration}

The BMS camera is used to make measurements of features on the road. The camera calibration is the process of establishing the transformation of points in the image into the corresponding points on the road surface. In the general case, camera calibration is a difficult problem. The calibration process can be greatly simplified for the case where the camera is fixed on the vehicle and is pointed relatively straight down on the road surface. The picture in Figure 6 shows how the camera is mounted to the vehicle for the tests conducted in this report.

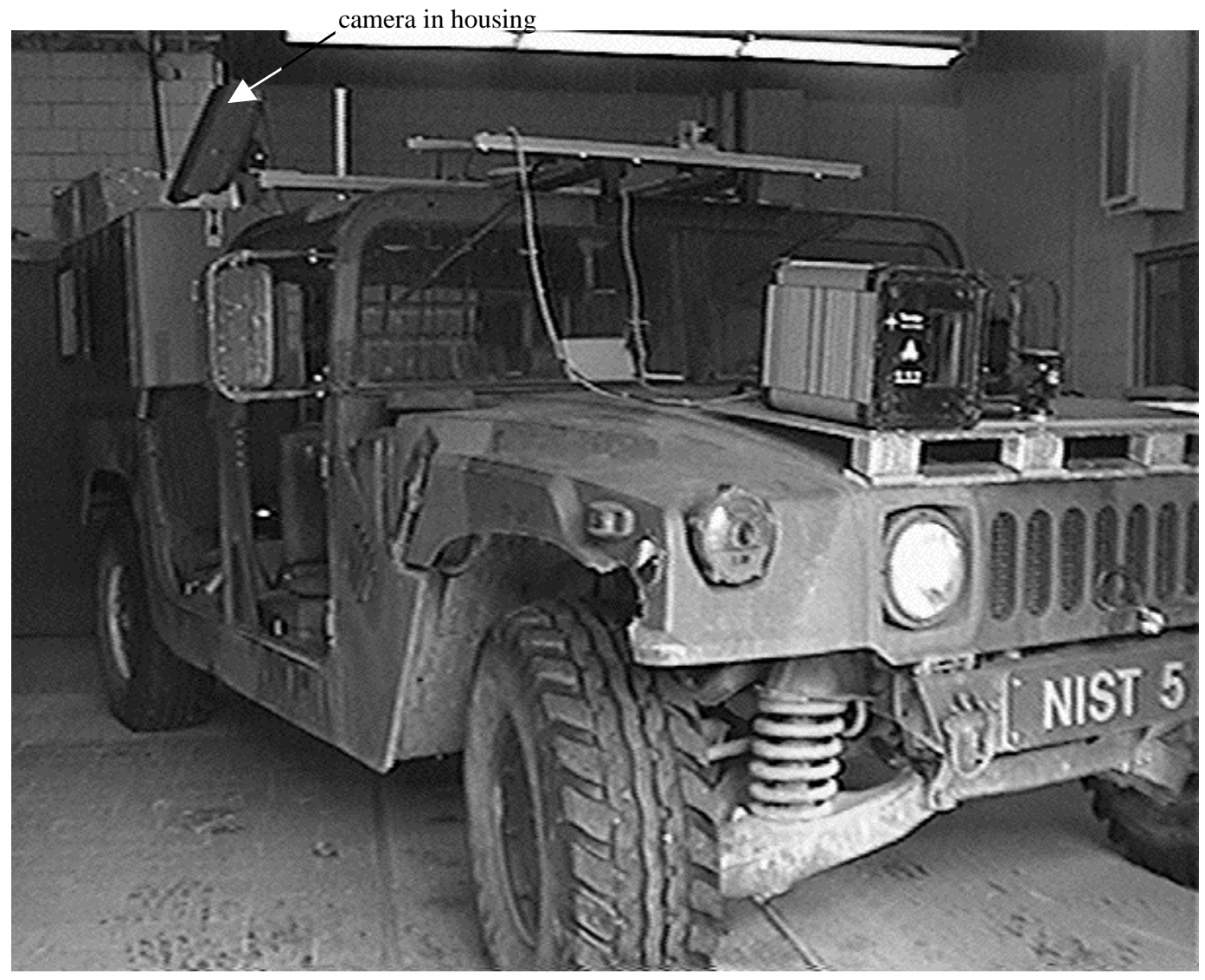

Figure 6. Picture of downward pointing camera mounted on side of vehicle.

The calibration process is divided into two phases. In the first phase, a look-up table is built that defines a transformation from points in the image plane, expressed in pixel coordinates, into 
points on a local coordinate system on the road surface, expressed in millimeters. In the second phase of calibration, the transformation between the local road-surface coordinate system and the GPS coordinates of the vehicle is established. Once the calibration is completed, a routine can be called which transforms $(\mathrm{i}, \mathrm{j})$ camera points into (North, East) GPS points.

\subsubsection{Local Road-Surface Calibration}

The calibration for the local road surface establishes a transformation between image points and road-surface points. A simple calibration is a scale factor that converts pixels into millimeters. A single scale factor does not accurately model the transformation from image coordinates to world coordinates. A lookup table calibration is used in the BMS to store scale factors throughout the image plane. The calibration is good for a single camera pose (position and orientation) relative to the road surface. The accuracy of the calibration depends on the fact that the camera pose and the road-surface orientation do not vary significantly. Besides camera pose, other variables affect the calibration process. One variable is the lens. A wide-angle lens will provide a greater coverage but will decrease the resolution, thereby changing the number of millimeters per pixel (i.e., the scale factor). A narrow-angle lens has the opposite effect. Another variable is the image format. When collecting data with the digital recorder, the user can select the number of rows or columns of an image to save. For example, in order to save disk space, every other row of the image may be skipped. In a full image, the 480 pixels in a column may span approximately $1.25 \mathrm{~m}$ on the road surface. Skipping every other row results in 240 pixels spanning $1.25 \mathrm{~m}$, thereby changing the calibration. In summary, the calibration must be performed whenever the camera pose, lens, or image digitization format is changed.

Figure 7 shows a picture of the target used to calibrate the side-looking camera. The target consists of a large, flat sheet that can be rolled out to accommodate varying fields-of-view. Since the sheet is very thin, the surface beneath is also effectively calibrated once the calibration is complete. The calibration target has a constant offset between control points, but the camera pose, lens, and image format distorts the offset. An image processing program, written in Khoros $^{3}$, computes the location of the centroids of each control point in the image. Figure 7 shows the output of the program. A white boundary around the perimeter of a control point indicates the centroid was successfully detected. The output of the Khoros program is an ASCII file containing the locations of the centroid of each control point.

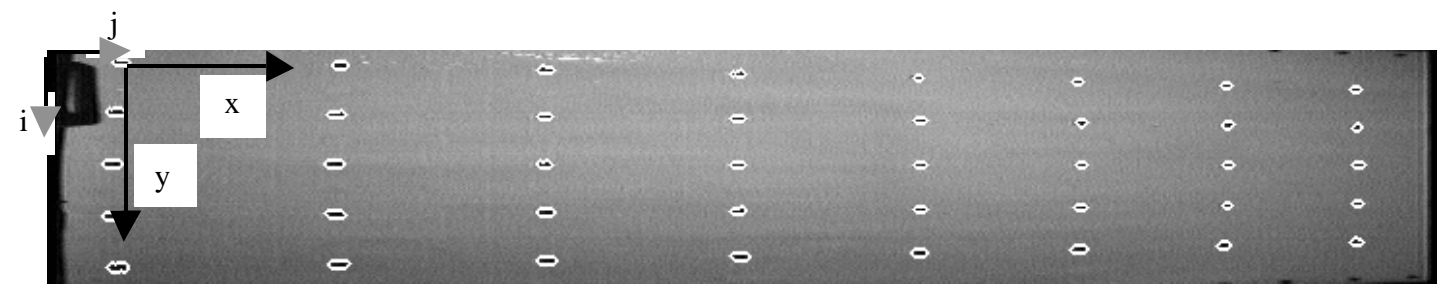

Figure 7. Calibration target is used to define a local road-surface origin $(x, y)$ at top left control point.

The next step is to identify the control points in the image that correspond with the control points on the calibration target. A C program is used for this step. Figure 7 shows the two coordinate systems used for this step. The origin of the image frame is at the top, right portion of the image 
(arrows labeled $\mathrm{i}$ and $\mathrm{j}$ ). The origin of a local road-surface coordinate system is at the top, right control point (arrows labeled $\mathrm{x}$ and $\mathrm{y}$ ). The program automatically matches centroids in the image with control points on the calibration target. It then assigns offsets in the $\mathrm{x}$ and $\mathrm{y}$ directions (relative to the local road-surface coordinate system) to each centroid based on the known offsets of the calibration target.

The last step in the local road-surface calibration is to build a lookup table that is used to transform pixels points into road-surface points. The lookup table, a $\mathrm{C}$ data structure, is created each time the system is initialized. Each node in the data structure contains the pixel coordinates of a control point in the image, the $(\mathrm{x}, \mathrm{y})$ coordinates of the corresponding control point in the local road surface coordinate system, and the coefficients for interpolating between pixels in the horizontal and vertical directions. Once the lookup table is created, a lookup routine is called whenever there is a need to transform an image point into a road-surface point. The lookup routine searches the data structure to isolate the bounding region containing the pixel and then uses linear interpolation to transform the point onto the road surface.

The camera calibration technique was evaluated to determine the FOV and the resolution in transforming image points to road-surface points. Table 2 summarizes certain attributes of the camera calibration performed for this report. Cam Ht is the height of camera (in meters) above the road (measured by hand). Lens is the lens focal length (in $\mathrm{mm}$ ) obtained from the lens body. Lat (perpendicular to vehicle travel) and Long (parallel to vehicle travel) FOV are the maximum coverage of the camera field-of-view expressed in meters. This is obtained using the calibration lookup table. Max and Min Res are the distances (in $\mathrm{mm}$ ) on the road surface subtended by a pixel in the lateral direction (along the $\mathrm{x}$ axis in Figure 7). This varies with pixel location. This is obtained using the lookup table.

\begin{tabular}{|c|c|c|c|c|c|}
\hline Cam Ht $\mathrm{m}$ & Lens $\mathrm{mm}$ & Lat FOV m & Long FOV m & Max Res mm & Min Res mm \\
\hline 1.375 & 6 & 1.7 & 1.25 & 1.86 & 3.28 \\
\hline
\end{tabular}

Table 2. Camera calibration attributes.

\subsubsection{Camera to GPS Calibration}

To complete the camera calibration, it is necessary to derive the transformation from the local road-surface coordinate system into the GPS coordinate system. Figure 8 shows some relevant coordinate systems used for this camera calibration. 
niu, veh, cam and gps_ant are rigidly attached to vehicle.

niu and veh xy planes are coplanar with axles.

cam xy plane is coplanar with road.
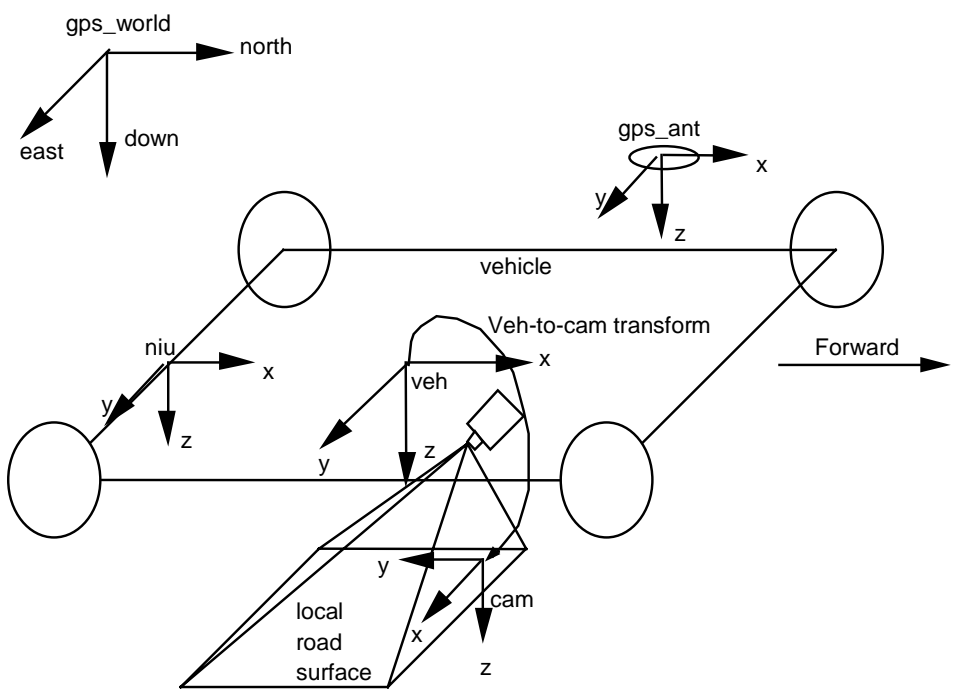

Figure 8. Vehicle coordinate systems describing camera-to-GPS calibration.

The BMS obtains the location of the vehicle whenever a camera image is taken. The vehicle location, shown as the coordinate system (CS) labeled veh, is relative to the gps_world CS. The camera-to-GPS calibration determines the transformation from the veh CS to the cam CS (labeled veh-to-cam transform in Figure 8). The $\mathrm{x}$ and $\mathrm{y}$ axes of the cam CS are identical to the $\mathrm{x}$ and y axes of the local road-surface CS shown in Figure 7. The veh-to-cam transformation depends on the position of the camera on the vehicle and the orientation of the camera with respect to the vehicle forward axis. Note that the transformation does not define the position and orientation of the camera on the vehicle. Once the veh-to-cam transformation is known, any pixel in the camera can be transformed back to the vehicle and then into GPS coordinates.

Computing the veh-to-cam transformation requires that two points on the road be surveyed manually using a GPS antenna. The position portion of the transformation only requires one point, $\mathbf{f}$, shown in Figure 9. 


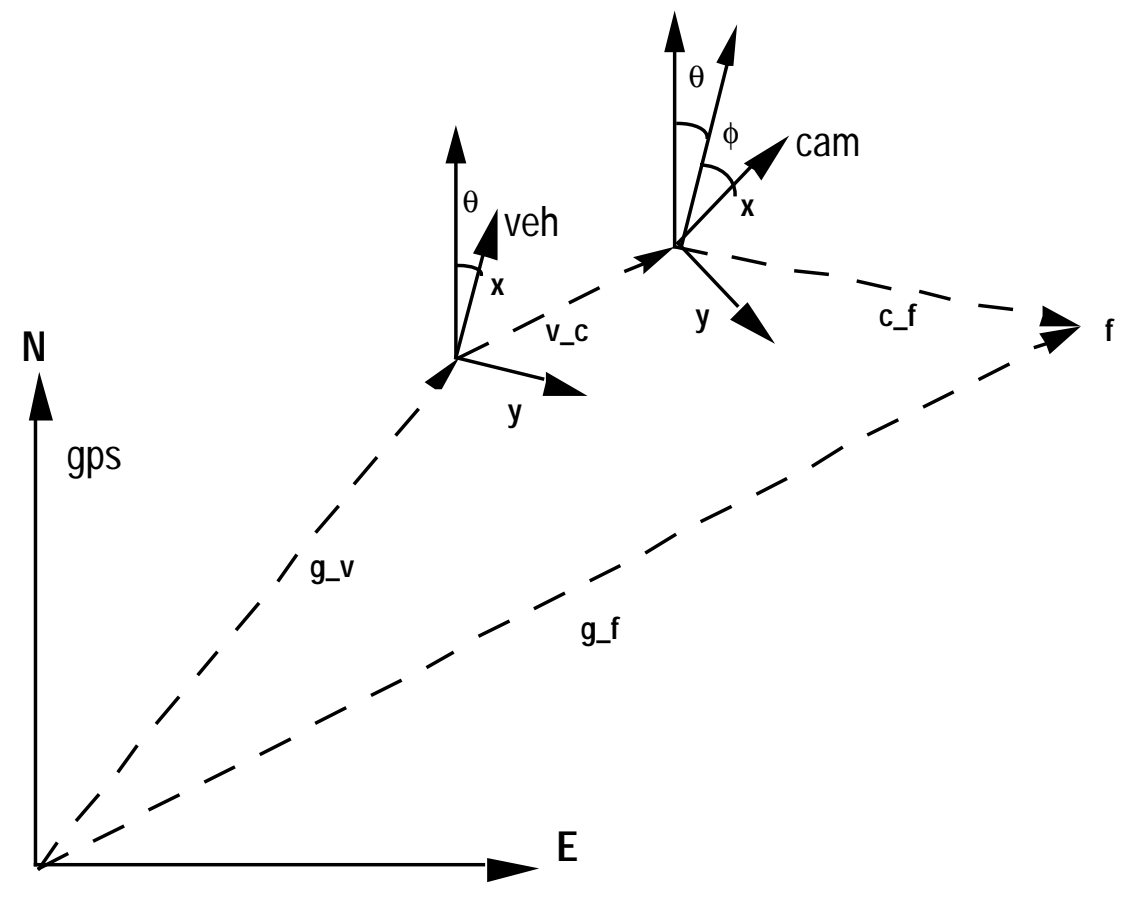

Figure 9. Derivation of position transformation between the local road surface and the GPS coordinate systems.

Figure 9 shows a top view of the coordinate systems shown in Figure 8. Below are the definitions of the systems.

1. gps - the world CS as measured by the orbiting GPS satellites.

2. veh - a CS centered within the vehicle. The output of the GPS is the location of the veh CS with respect to the GPS CS.

3. cam - a CS in the camera field-of-view whose origin is the local road-surface origin shown in Figure 7 rotated so the $\mathbf{x}$ vector in the vehicle forward direction. The output of the lookup function is expressed as a vector in the cam CS.

The following vectors are shown in Figure 9.

1. $\mathbf{g} \_\mathbf{v}$ - the position of the vehicle in GPS coordinates. This is given by the GPS.

2. $\quad$ __f - the position of the point $\mathbf{f}$ in the cam CS. c_f $\mathbf{f}=\operatorname{lookup}(i, j)$ where $(i, j)$ is the pixel coordinates of point $\mathbf{f}$ lookup is a routine that returns the GPS coordinates of an image point $(\mathrm{i}, \mathrm{j})$. The point is selected manually while viewing the image on a screen.

3. $\mathbf{g} \_\mathbf{f}$ - the position of point $\mathbf{f}$ derived by manually surveying the point using a GPS antenna above the point.

4. $\mathbf{v} \_\mathbf{c}$ - The position of the cam CS with respect to the veh CS. This vector is the translation portion of the GPS calibration, which is unknown and is the value to derive.

The following angles are also shown in Figure 9:

1. $\theta$ - the heading of the vehicle. This is derived from the GPS system and is with respect to North.

2. $\phi$ - the angle of the cam CS with respect to the veh $\mathbf{x}$ axis. This angle is the orientation portion of the GPS calibration, which is unknown and will be derived later. 
The solution for the veh-to-cam transformation does not require the roll and pitch angles of the vehicle. This is acceptable since the roll and pitch of the cam CS (i.e., the local road surface) closely parallels the veh CS.

As can be seen from Figure 9, the vector $\mathbf{g} \mathbf{f}$ can be written as:

$$
\mathbf{g} \_\mathbf{f}=\operatorname{trans}\left(\mathbf{g} \_\mathbf{v}\right)+\operatorname{rotz}(\theta) \operatorname{trans}\left(\mathbf{v} \_\mathbf{c}\right)+\operatorname{rotz}(\theta+\phi) \operatorname{trans}\left(\mathbf{c} \_\mathbf{f}\right)
$$

[Note: The function rotz (angle) defines a rotation of angle about the $\mathrm{z}$ axis (positive direction of $\mathrm{z}$ is out from the page) and the function trans(vector) defines a translation along the vector (the magnitude of the translation is equal to the magnitude of the vector).] The translation vector $\mathbf{v} \_\mathbf{c}$ can be derived from (3-4) as follows:

$$
\mathbf{v} \_\mathbf{c}=\operatorname{rotz}(-\theta)\left[\mathbf{g} \_\mathbf{f}-\operatorname{rotz}(\theta+\phi) \operatorname{trans}\left(\mathbf{c} \_\mathbf{f}\right)-\operatorname{trans}\left(\mathbf{g} \_\mathbf{v}\right)\right]
$$

The orientation portion of the camera calibration does not require the roll and pitch, only the angle between the forward axes of the cam CS and the veh CS. The camera orientation can be derived using points $\mathbf{b}$ and $\mathbf{f}$ in Figure 10.

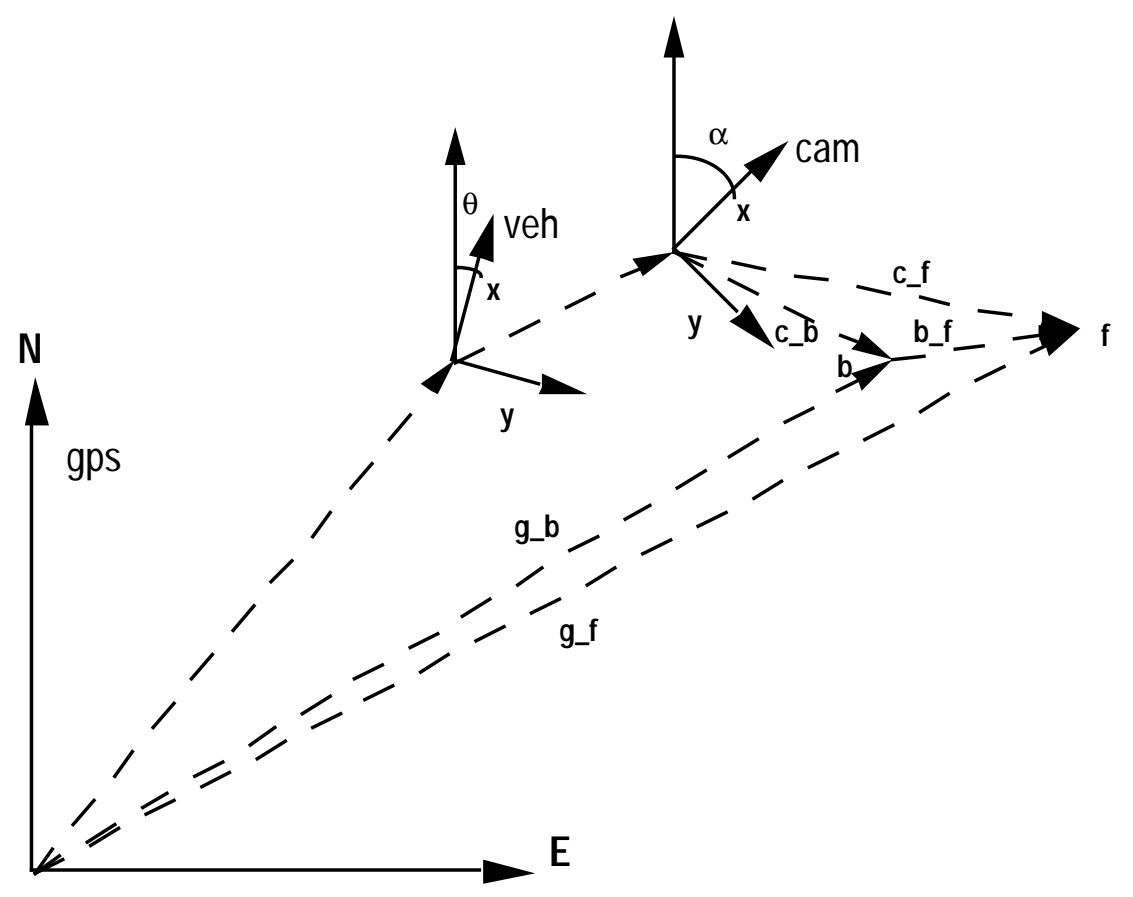

Figure 10. Two points are required to derive the rotation transformation between the local road surface and the GPS coordinate systems.

The new vectors in Figure 10 are: 
1. $\mathbf{c} \_\mathbf{b}$ - the position of the point $\mathbf{b}$ in the cam CS. $\mathbf{c} \_\mathbf{b}=\operatorname{lookup}(\mathrm{i} . \mathrm{j})$ where $(\mathrm{i}, \mathrm{j})$ is the pixel coordinates of point $\mathbf{b}$. The point is selected manually while viewing the image on a screen.

2. $\mathbf{g} \_\mathbf{b}$ - the position of point $\mathbf{b}$ derived by manually surveying the point using a GPS antenna above the point.

3. b_f - the vector from point $\mathbf{b}$ to point $\mathbf{f}$. The vector can be derived in either the GPS or cam coordinate systems.

The new angle in Figure 10 is:

1. $\alpha$ - the angle of the cam CS with respect to North. This angle, as shown in Figure 9, equals $\theta$ plus $\phi$.

This leads to the following equation to be solved:

$$
\phi=\alpha-\theta
$$

Figure 11 shows how the difference in the vectors as expressed in each coordinate system is used to derive the rotation portion of the calibration.

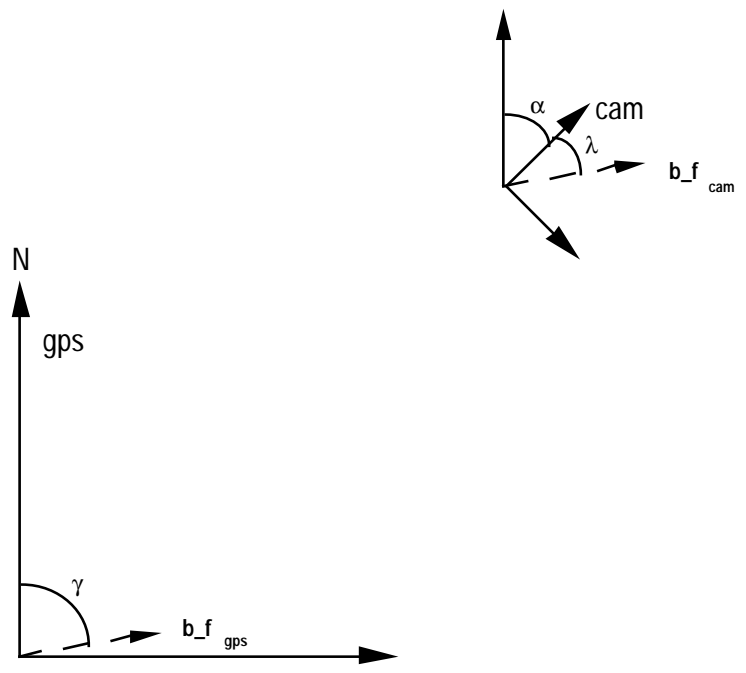

Figure 11. Derivation of rotation transformation between camera and GPS coordinate systems.

The following vectors shown in Figure 11 can be derived using vector addition (see also Figure 10):

$$
\begin{aligned}
& \text { b_f } \mathbf{f}_{\text {cam }}=\mathbf{c} \_\mathbf{f}-\mathbf{c} \_\mathbf{b} \\
& \mathbf{b \_ f} \mathbf{f}_{\mathrm{gps}}=\mathbf{g \_ f}-\mathbf{g} \_\mathbf{b}
\end{aligned}
$$

The equations in ( 3-7 ) can be used to define two additional angles: 
1. $\gamma$ - the angle of $\mathbf{b}_{-} \mathbf{f}_{\mathrm{gps}}$ with respect to North.

2. $\lambda$ - the angle of $\mathbf{b}_{-} \mathbf{f}_{\text {cam }}$ with respect to the forward axis of the cam CS.

These angles are derived by:

$$
\begin{aligned}
& \gamma=\pi / 2-\arctan \left(\mathbf{b}_{-} \mathbf{f}_{\text {gps }}\right) \\
& \lambda=\pi / 2-\arctan \left(\mathbf{b}_{-} \mathbf{f}_{\text {cam }}\right)
\end{aligned}
$$

As can be seen in Figure 11, the angle $\lambda$ represents the angle of $\mathbf{b}_{-} \mathbf{f}_{\text {cam }}$ as seen through the camera. The fact this differs from $\gamma$, the angle of the $\mathbf{b}_{-} \mathbf{f}_{\mathrm{gps}}$ on the road, is the result of the rotation of the cam coordinate system by the angle $\alpha$. Therefore,

$$
\gamma=\alpha+\lambda
$$

Given the known values $\gamma, \lambda, \theta$ and equation ( 3-6 ) yields:

$$
\begin{aligned}
\phi & =\gamma-\lambda-\theta \\
& =\arctan \left(\mathbf{b}_{\mathbf{f}} \mathbf{f a m}_{\mathrm{cam}}\right)-\arctan \left(\mathbf{b}_{\mathbf{f}} \mathbf{f}_{\mathrm{gps}}\right)-\theta
\end{aligned}
$$

Once the calibration has produced the position and orientation parameters, any point $\mathbf{c} \_\mathbf{f}=$ lookup(i,j), can be transformed into Northing and Easting using equation (3-4 ).

\subsection{Video Digital Recording System}

The components of the Video Digital Recording System are shown in Figure 12. They consist of a camera, a Silicon Graphics Inc. O2 computer, and a RAID (Redundant Array of Inexpensive Disks) storage device. A picture of the system is shown in Figure 13. The $\mathrm{O} 2$ is used to digitize video, read GPS/INS data, read data from a system under evaluation, and store all data onto the RAID. The RAID consists of three 9-MB hard disks that operate in parallel but appear as a single volume in the file system. The $\mathrm{O} 2$ is ruggedized to withstand shock and vibration disturbances when the vehicle is driven off-road. 


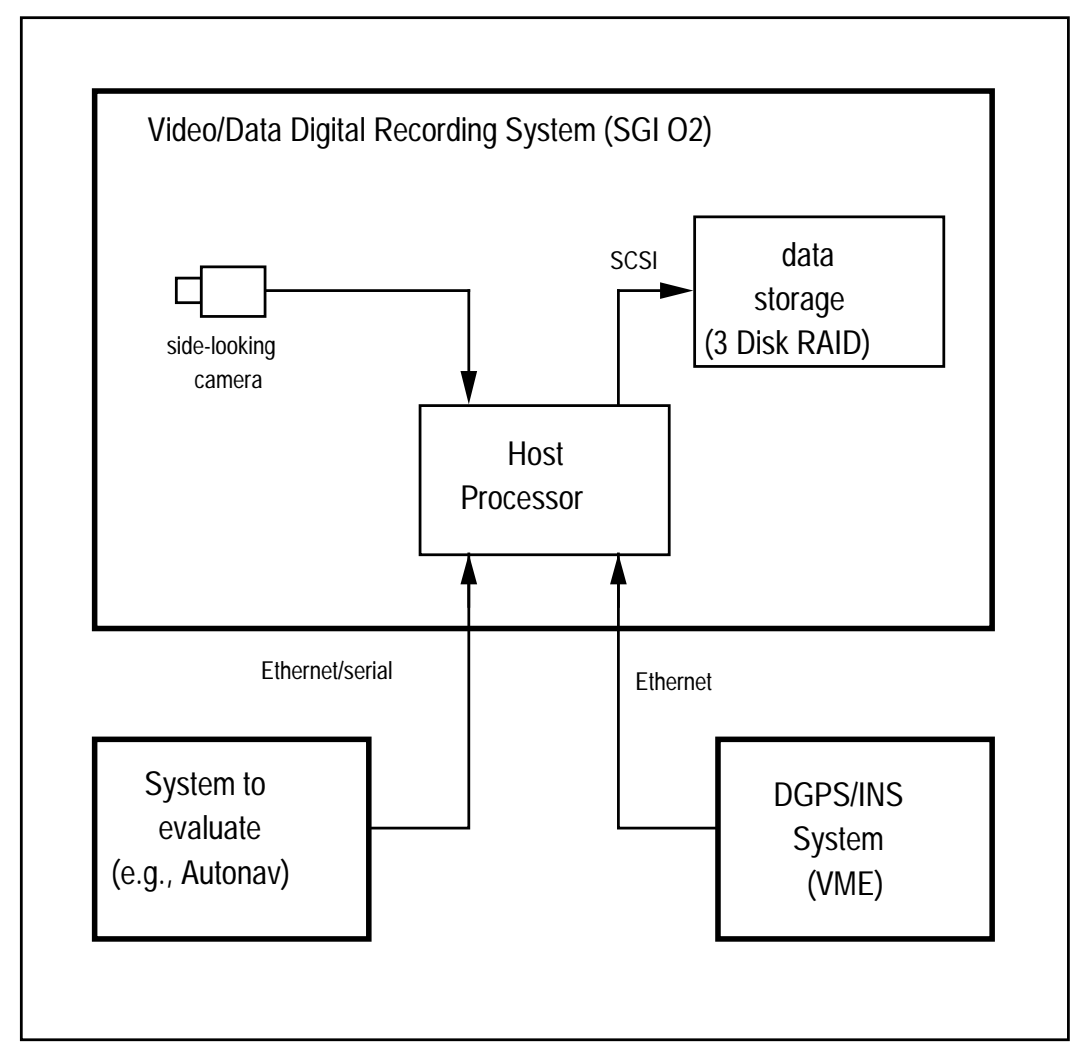

Figure 12. Components of the Video Digital Recording System.

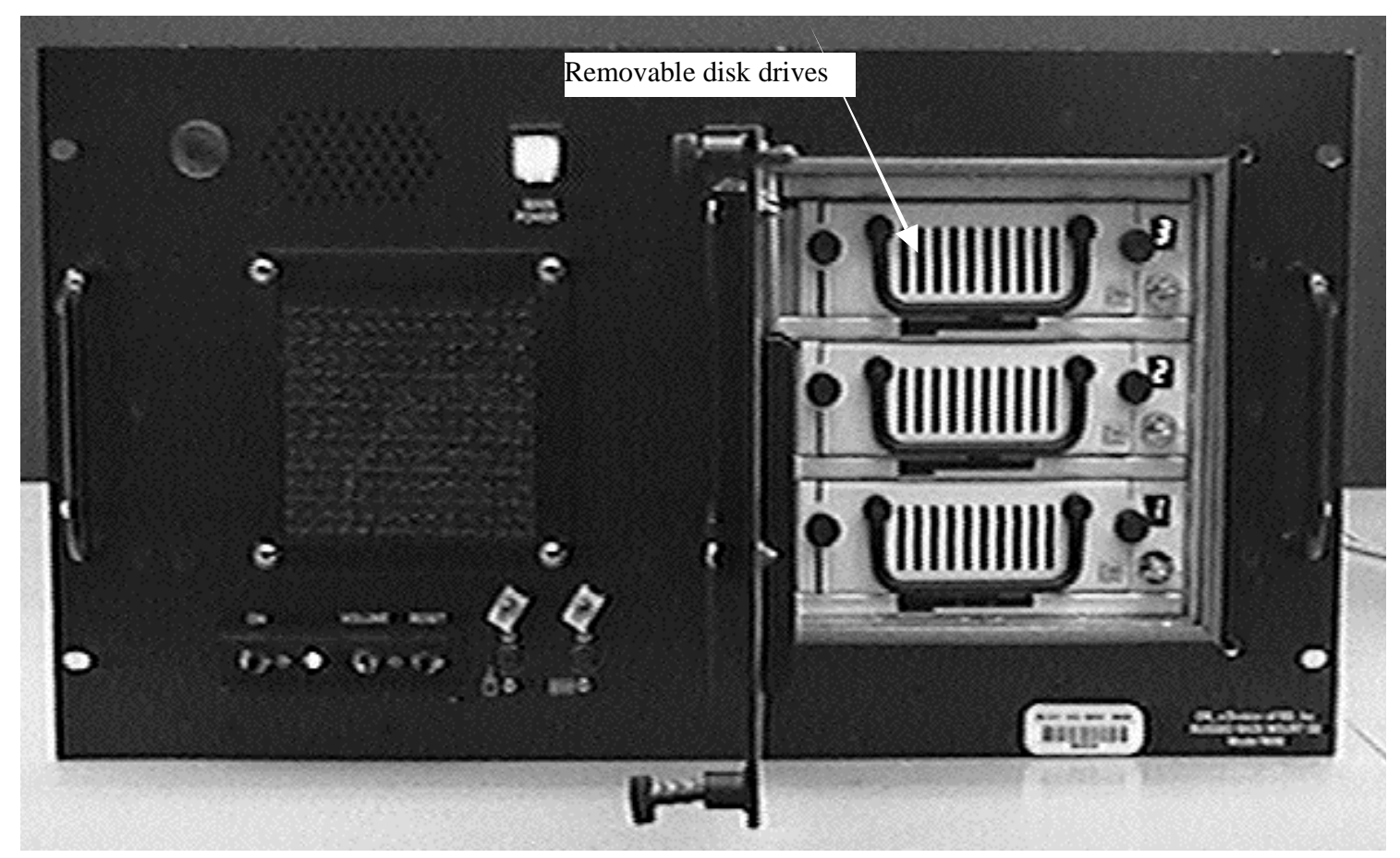

Figure 13. Picture of the BMS Video/Data Digital Recording System. 
Data collection consists of recording video and GPS/INS data simultaneously. A single C routine is used to initialize the $\mathrm{O} 2$ video input, to read in each image, to read in the current GPS/INS data, to format the image, and to write the video and GPS/INS data to disk.

The maximum recording bandwidth of the $\mathrm{O} 2$ is achieved by routing the video directly to a disk buffer without accessing individual pixels. Any processor access to video memory, for example to examine some pixel value, significantly reduces the recording bandwidth. The $\mathrm{O} 2$ is able to record color video at $640 \times 480$ pixels at 30 frames per second, or at 320x 240 pixels at 60 fields per second. Occasional dropouts may occur. The color format uses 2 bytes per pixel. The resultant bandwidth is $\left(640 \times 480 \times 2 \times 30 / 1024^{\wedge} 2\right) \cdot 17.6 \mathrm{Mb} / \mathrm{s}$. 


\section{Evaluation of Baseline Measurement System}

Several tests were conducted to evaluate the BMS and to show how the BMS might be used to measure the performance of a countermeasure system. Below is a summary of some factors that affect the accuracy of the BMS. Later in this section are the results of several tests conducted to validate the BMS and to evaluate its performance.

\section{GPS Receiver}

Radio link dropout, signal multi-path, satellite geometry, and satellite-lock (i.e., the satellite is no longer visible due to trees, building, etc.) all affect GPS accuracy. During dynamic measurements (i.e., while the vehicle is moving), delays in processing and communications are a significant source of error. During these tests, we relied on the real-time capabilities of the GPS without any corrections or post-processing of the data.

Inertial Navigation System

In order to increase the update rate of the vehicle position measurement, only the INS gyros and odometry are used. The drift in this mode of operation is primarily due to wheel slippage and is generally a function of distance traveled. The drift is reduced whenever the GPS updates are received. Thus, the predominate effects of INS drift are confined to the periods between the half second GPS updates and when the GPS loses lock with the satellites.

\section{Kalman Filter Algorithm}

The Kalman Filter combines data between the GPS and the INS, so uncertainties will depend to a large part on the uncertainties contributed by these components. In addition, a small error has been identified due to the differences in the latencies of the GPS and INS. An algorithm has been developed that appears to correct for these errors but the algorithm was not completely implemented in time for these tests.

\section{Camera Calibration}

The camera calibration transforms pixel coordinates into GPS coordinates. There are two parts to the transformation. The first part transforms pixels coordinates into $\mathrm{x}, \mathrm{y}$ coordinates (in $\mathrm{mm}$ ) in a local road-surface coordinate system (i.e., the road in the camera FOV). The uncertainties in this part of the calibration are due to the resolution of the camera, the distortion of the lens, the camera orientation with respect to the road, and the density of the calibration target. The resolution of the camera falls in the $2 \mathrm{~mm}$ to $3 \mathrm{~mm}$ range. The accuracy of this transformation is difficult to isolate due to the large number of factors, but it can be assumed the uncertainty is bounded from below by the resolution. The second part of the transformation converts local road-surface coordinates into GPS coordinates. Since the GPS receivers are used to establish this transformation, the accuracy of this transformation depends on the uncertainties of the GPS.

\section{Test Procedures}

The tests consist of running the vehicle over various parts of the NIST grounds and collecting data using the BMS. The data for each run consists of video recorded at $60 \mathrm{~Hz}$. and, for each video frame, the corresponding data from the GPS, INS and the Kalman filter. The video data and the position (or navigation) data are stored in individual files. A naming convention 
simplifies keeping track of the video and data. Table 3 shows the video file names. The file name contains the date and time the video was collected, the format (in this case $60 \mathrm{~Hz}$ field video) and the dimensions of each frame. The names of the position data files corresponding to the video are shown in Table 4. By looking at the "time" field of the file name, one can see that the total time required to collect six runs of data was approximately $13 \mathrm{~min}$ (from hr-mm-ss of 10-39-30 to 10-52-56). This illustrates the ease in which data can be collected using the BMS.

\begin{tabular}{|l|l|}
\hline \multicolumn{1}{|c|}{ Size (bytes) } & File Name (_date_time_hzxformat_cols $x$ row) \\
\hline $196,455,600$ & -04-03-98_10-39-30_60xFI_720x121.raw \\
\hline $647,388,720$ & -04-03-98_10-41-40_60xFI_720x121.raw \\
\hline $621,078,480$ & -04-03-98_10-44-28_60xFI_720x121.raw \\
\hline $664,638,480$ & 04-03-98_10-47-46_60xFI_720x121.raw \\
\hline $616,809,600$ & 04-03-98_10-50-17_60xFI_720x121.raw \\
\hline $1,259,493,840$ & -04-03-98_10-52-56_60xFI_720x121.raw \\
\hline
\end{tabular}

Table 3. Video files recorded for test.

\begin{tabular}{|l|l|}
\hline \multicolumn{1}{|c|}{ Size (bytes) } & File Name (_date_time_hzxformat_colsxrow) \\
\hline 505120 & $-04-03-98 \_10-39-30 . n a v$ \\
\hline 1664544 & $-04-03-98 \_10-41-40 . n a v$ \\
\hline 1596896 & $-04-03-98 \_10-44-28 . n a v$ \\
\hline 1708896 & -04-03-98_10-47-46.nav \\
\hline 1585920 & 04-03-98_10-50-17.nav \\
\hline 3238368 & -04-03-98_10-52-56.nav \\
\hline
\end{tabular}

Table 4. Navigation data files corresponding to video files in Table 3.

A reference for comparison was established by inserting nails into the road asphalt. The nails were surveyed manually using the GPS. The survey involved long-term averaging and the use of carrier phase corrections. The uncertainties are believed to lie in the $1 \mathrm{~cm}$ to $2 \mathrm{~cm}$ range. The nails were highlighted, see Figure 14, with black and white paint. The highlighted nails make it easy for the analyst to select the nail in the image. The nails provide a reference point for evaluating the BMS. At any video frame where the nail is visible, the nail can be selected and transformed into GPS space. The coordinates of the nail acquired visually can be compared to the manual survey measurements to determine uncertainties in the BMS. The remainder of this section covers the tests and the results.

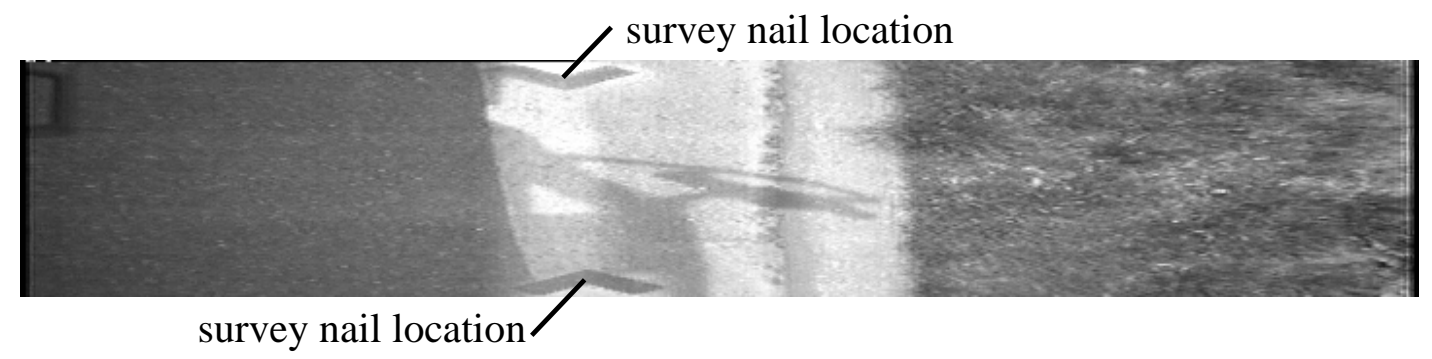

Figure 14. A single frame recorded by BMS showing two survey nails highlighted using black and white paint. 


\subsection{Verification of Camera Calibration}

The goal of this test was to establish the correctness of the camera calibration software. The vehicle was driven to a point where a survey nail was visible in the video. The BMS recorder was turned on and the vehicle was driven through a half figure eight and stopped facing in the opposite direction with the survey nail again visible in the video. Figure 15 shows the trajectory of the vehicle and the locations of the nail acquired visually using the BMS. The unfiltered_gps line is the raw GPS data at half-second updates, the filtered_gps is the output of the Kalman filter, and the ins is the output of the MAPS.

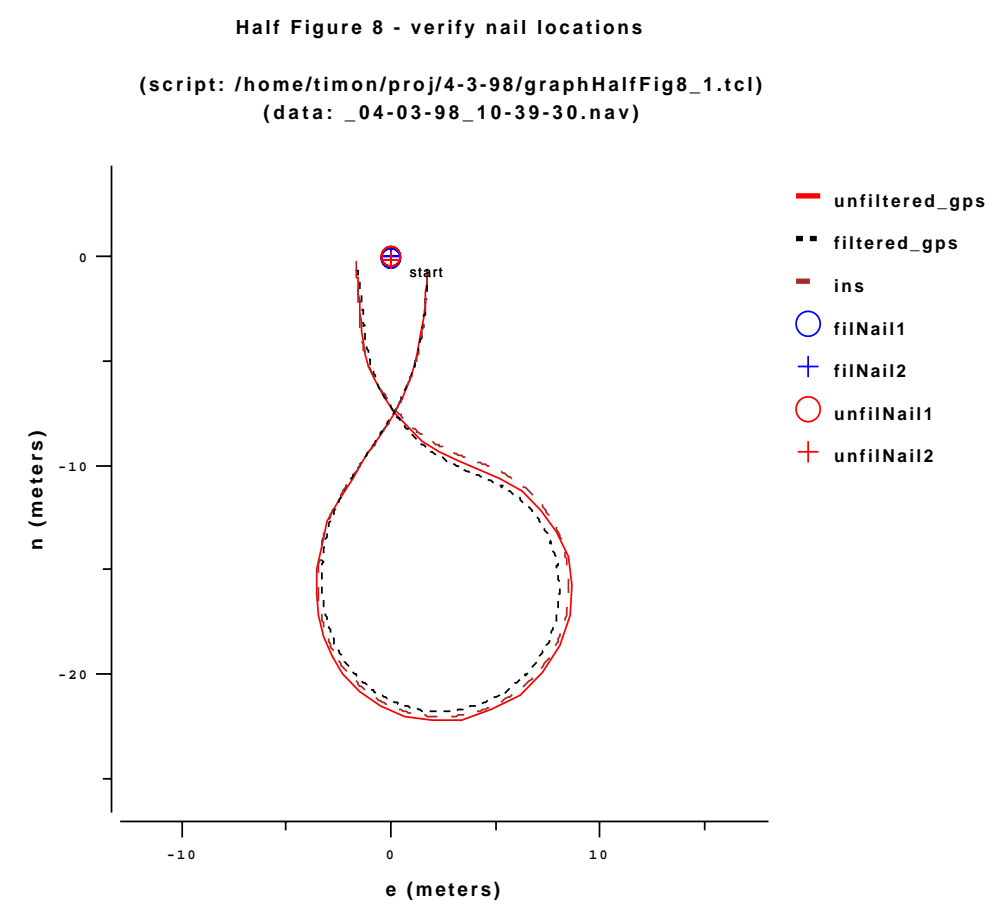

Figure 15. Vehicle driven along a half figure-eight trajectory along with survey nails acquired using video at beginning and end (see also Figure 16).

Acquiring the nail in both direction exercises the calibration transformations from the camera's FOV to GPS space. If the calibration parameters and the transformations are correct, the nail should align at the beginning and at the end, and should coincide with the surveyed coordinates of the nail. Figure 16, a zoomed in view of the nails in Figure 15, shows the coordinates of the nail acquired in the first (legend titles ending in 1) and last (legend titles ending in 2) video frame. The coordinates of the nail are calculated using the vehicle's filtered and unfiltered GPS coordinates. The origin of the graph is the surveyed location of the nail. Two of the points align 
very closely with the origin. The locations of the nails and their distance from the surveyed nails are listed in Table 5.

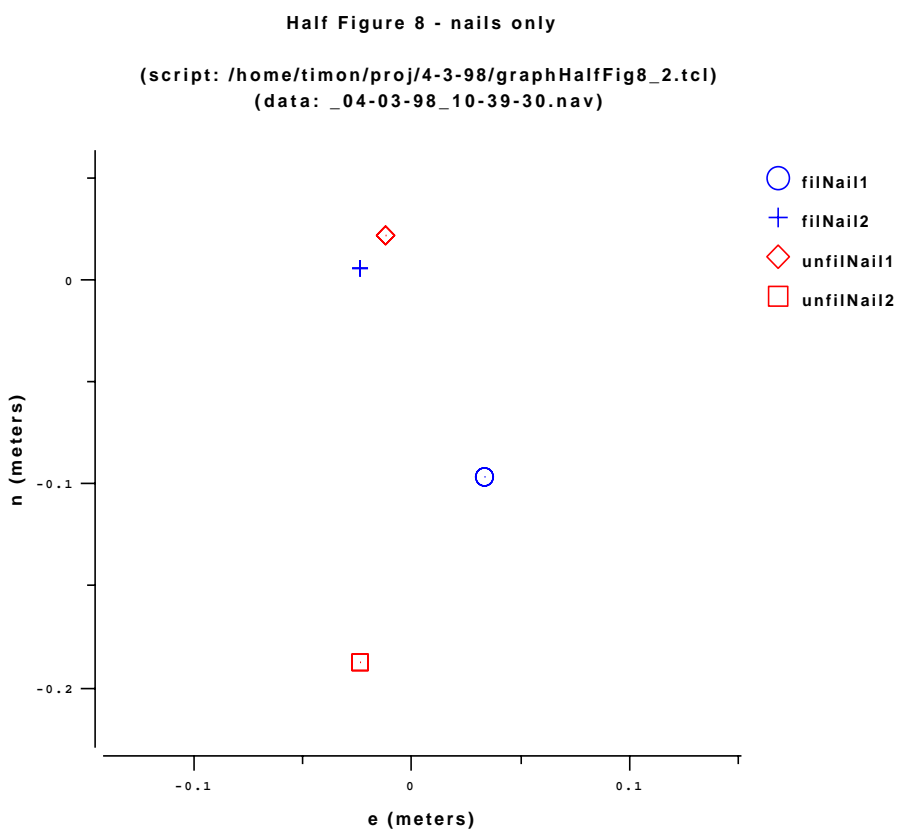

Figure 16. Filtered and unfiltered (raw) GPS coordinates of nail at beginning and end of half figure 8 trajectory.

\begin{tabular}{|l|r|r|r|}
\hline \multicolumn{1}{|c|}{ Data Point Name } & North $(\mathrm{m})$ & \multicolumn{1}{c|}{ East $(\mathrm{m})$} & Distance From Origin $(\mathrm{cm})$ \\
\hline filNailN1 & -0.096 & 0.033 & 10.2 \\
\hline filNailN2 & 0.005 & -0.023 & 2.4 \\
\hline unfilNailN1 & 0.021 & -0.011 & 2.4 \\
\hline unfilNailN2 & -0.187 & -0.023 & 18.8 \\
\hline
\end{tabular}

Table 5. Values of visually acquired nails and their distance from the origin (i.e., the known survey location of the nail).

\subsection{Static Measurements}

This test seeks to establish the uncertainty of the BMS in measuring the position of a survey nail using the video system and the GPS while the vehicle is static (i.e., stationary). Several runs were made on the NIST grounds. Figure 17 shows the typical path in GPS space the vehicle took during a run. 


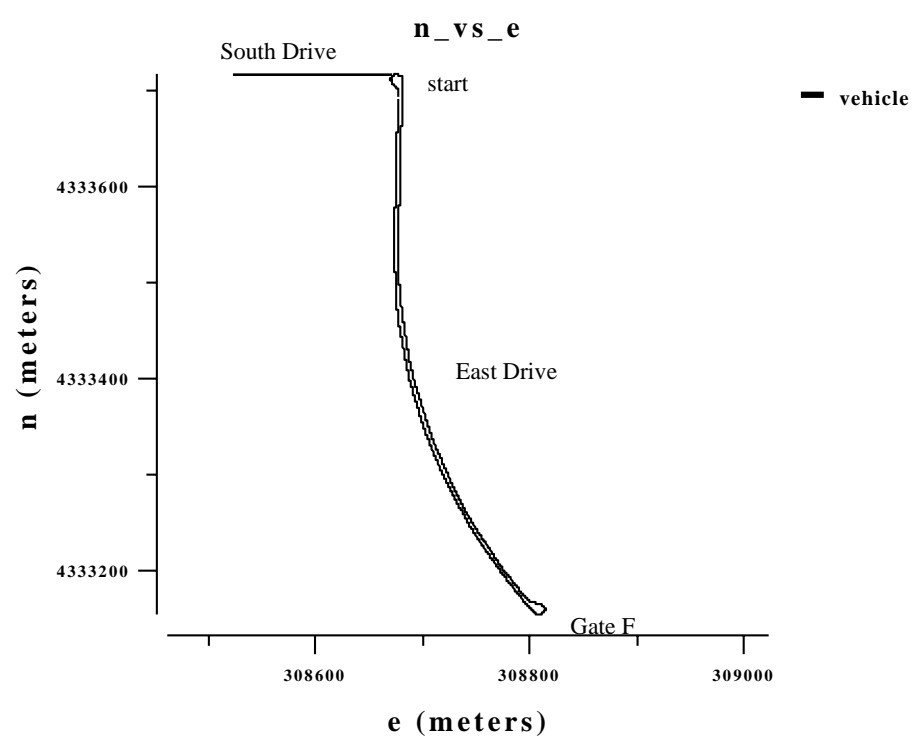

Figure 17. Trajectory of vehicle during test to acquire nail at beginning and end of run.

The survey nail was acquired visually at the beginning and end of each run. The location of the nail was obtained after the vehicle was stopped and in only one video frame. Figure 18 and Table 6 show the measured locations of the nail. Only runs where satellite-lock was maintained are included in this test. Again, the origin is the surveyed location of the nail. The coordinates of the visually surveyed nail are computed using the unfiltered (raw) GPS data in order to isolate the errors due to the INS and the Kalman filter. The majority of the points are close to the origin. It is unknown what caused one nail to appear $53.905 \mathrm{~cm}$ from the origin. The standard uncertainty (i.e., the standard deviation) over all the measurements in Table 6 is $+/-18 \mathrm{~cm}$. The expanded uncertainty ${ }^{4}$ (based upon a t-distribution and 95\% confidence level) is $+/-42 \mathrm{~cm}$. This is within the stated specifications of the manufacturer ${ }^{5}$ 


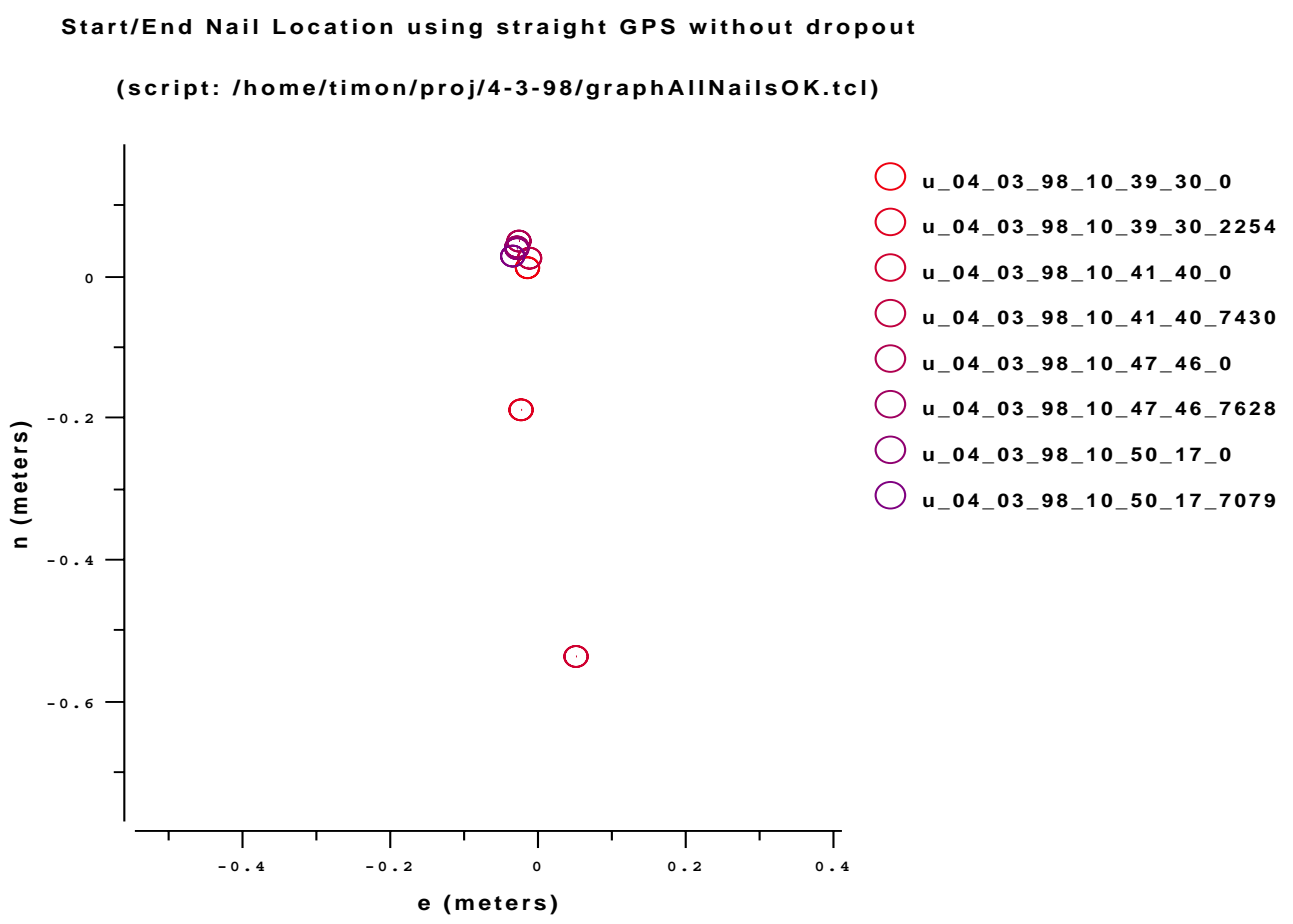

Figure 18. Results of visually acquiring survey nail at the beginning and end of several runs.

\begin{tabular}{|l|r|r|r|}
\hline \multicolumn{1}{|c|}{ Data Point Name } & North $(\mathrm{m})$ & \multicolumn{1}{c|}{ East $(\mathrm{m})$} & Distance From Origin $(\mathrm{cm})$ \\
\hline u_04_03_98_10_39_30_0 & 0.012 & -0.014 & 1.822 \\
\hline u_04_03_98_10_39_30_2254 & -0.187 & -0.024 & 18.887 \\
\hline u_04_03_98_10_41_40_0 & -0.537 & 0.052 & 53.905 \\
\hline u_04_03_98_10_41_40_7430 & 0.026 & -0.013 & 2.870 \\
\hline u_04_03_98_10_47_46_0 & 0.050 & -0.025 & 5.593 \\
\hline u_04_03_98_10_47_46_7628 & 0.041 & -0.028 & 4.946 \\
\hline u_04_03_98_10_50_17_0 & 0.040 & -0.029 & 4.905 \\
\hline u_04_03_98_10_50_17_7079 & 0.030 & -0.033 & 4.481 \\
\hline
\end{tabular}

Table 6. Visual-based measurements of nail (see Figure 18) with distance from manual surveyed coordinates of the nail (origin).

\subsection{Dynamic Measurements and Latency Calculation}

A dynamic GPS measurement, as opposed to a static measurement, is obtained while the vehicle is moving. Latency in obtaining the GPS measurement from the receiver is one source of uncertainty when making dynamic measurements. An approach to determine latency is to compare the coordinates of a survey nail obtained visually while driving against the coordinates of the survey nail obtained manually by survey.

To obtain a dynamic measurement, a survey nail was visually captured using the camera while the vehicle was driven along a road. Figure 19 shows only the section of the vehicle's trajectory as it drives by the surveyed nail. The vehicle's trajectory, the circles connected by lines, is 
labeled as filtered_gps. Each circle represents a Kalman filter update. The manually surveyed coordinates of the nail, the plus sign labeled surveyedNail, is shown at the origin. The nail acquired visually using the camera and GPS filtered data is shown as the circle labeled videoNail.

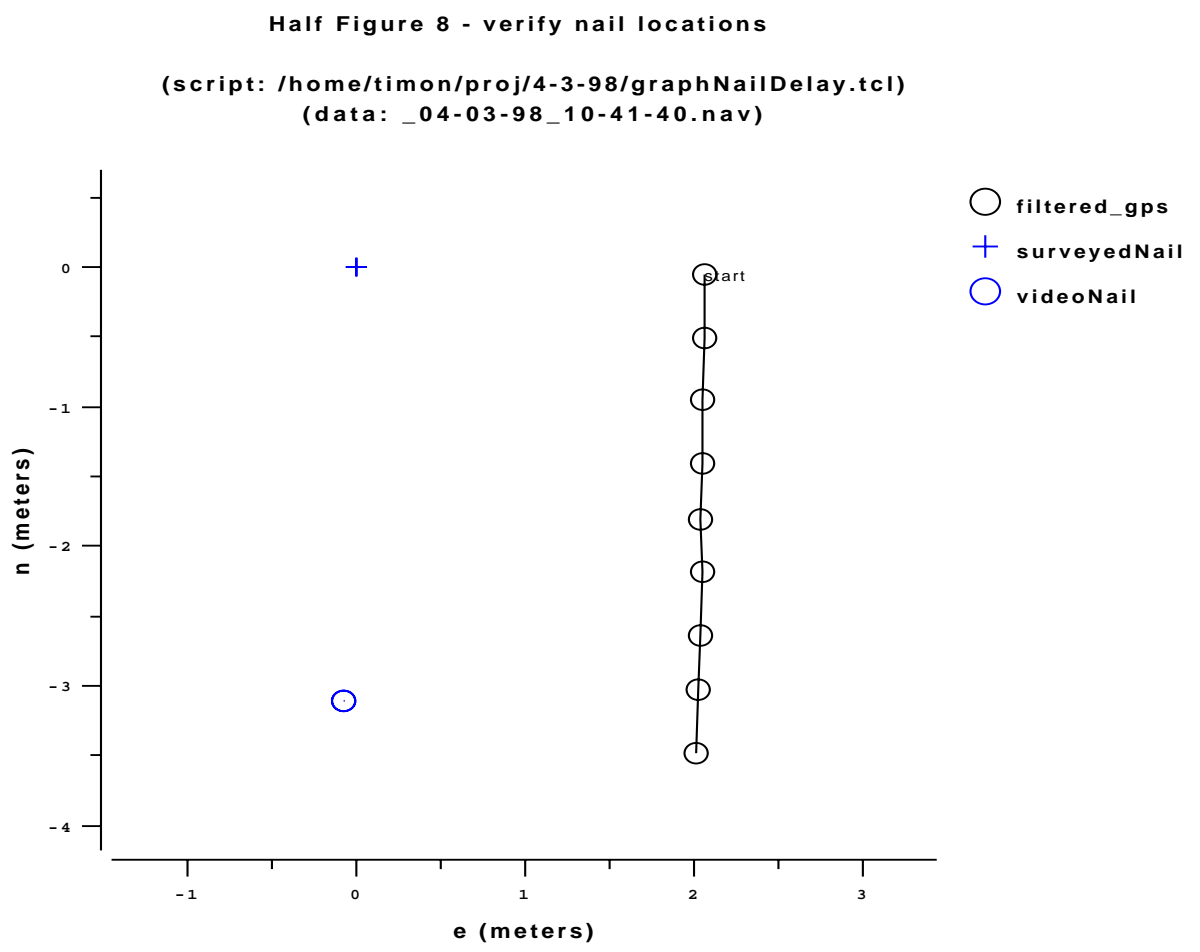

Figure 19. The error due to latency is shown in the discrepancy between the coordinates of the surveyed nail (surveyedNail) and the visually acquired nail (videoNail) as the vehicle (filtered_gps) drives by at $10.75 \mathrm{~m} / \mathrm{s}$.

Two methods are used to calculate the latency. One method uses the relationship velocity $=$ distance / time. Let distance equal the difference between the nail's surveyed coordinates and the nail's coordinates measured visually. Let velocity equal the speed given by the odometer (10.75 $\mathrm{m} / \mathrm{s}$ ). Solving for time in the velocity relationship yields a latency of $316 \mathrm{~ms}$.

To calculate the latency in a different manner, each point along the vehicle's trajectory is examined and the point falling closest to the survey nail is identified. For this data set, the vehicle passed closest to the nail's coordinates at GPS data record 837 (this data record corresponds with video frame 837, which is labeled "start" in Figure 19). The nail was visually located in video frame 852 (this corresponds to GPS data record 852). Each record is time stamped and subtracting the differences between the time stamps yields a latency of $300 \mathrm{~ms}$. This compares closely with the previously mentioned latency estimate of $316 \mathrm{~ms}$. These methods show how a latency calculation might be made but additional measurements need to be made prior to obtaining an uncertainty value for the estimate.

The GPS receiver manufacturer claims their fast algorithm for solving carrier phase GPS location produces a solution in less than $250 \mathrm{~ms}$. The additional latency measured also takes into account 
the delays in communications between the BMS and the GPS receiver, and any internal processing in the BMS.

A $300 \mathrm{~ms}$ latency increases the uncertainty in the vehicle's position proportional to velocity. For example, at $30 \mathrm{~m} / \mathrm{s}$ (roughly $60 \mathrm{miles} / \mathrm{hr}$ ), the latency increases the uncertainty by 9 meters. For longitudinal measurements, this uncertainty is significant. For lateral measurements, since the velocity approaching the lane marker is small, the uncertainty is smaller. One approach to reduce the uncertainty, not examined for this report, is by extrapolating vehicle position based on its previous trajectory. This partially depends on a small variance in latency, which again suggests additional measurements need to be made.

\subsection{Affects of GPS Dropout}

One of the main sources of GPS errors is the loss of satellite-lock. During two of the test runs, satellite loss occurred and the BMS relied on the INS for position measurement. Figure 20 shows a run starting at the intersection of East and South Dr. on the NIST grounds. The vehicle was driven onto Muddy Branch Rd., back into NIST, along Center Dr., and returned to the start location. To simplify reading the axis scales, the origin of the graph is offset from absolute GPS coordinates to the start location of the vehicle. The run took approximately $4.5 \mathrm{~min}$ and covered a distance of $2885 \mathrm{~m}$.

Example of gps dropout

(script: /home/timon/proj/4-3-98/graphDropout3.tcl)

(data : _04-03-98_10-52-56.nav)

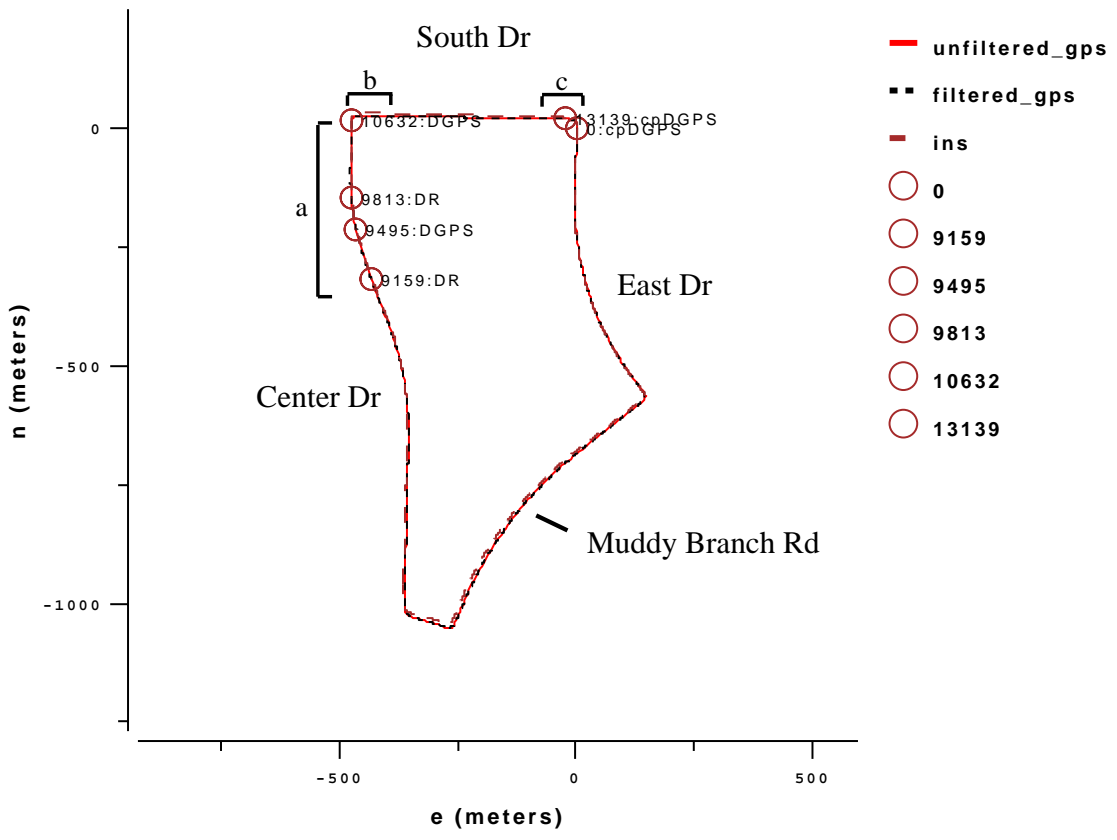

Figure 20. A run out the NIST gate onto Muddy Branch Rd and then back to the start showing mode changes in the navigation system (DR = DeadReckoning, DGPS = Differential GPS, cpDGPS = carrier phase DGPS). 
The circles indicate where the navigation system changed modes. Each circle is labeled with the new mode and the video frame number where the mode change occurred. At the start of the run, the navigation system was in carrier phase DGPS mode. This mode is sustained until the vehicle was driven beneath trees (at frame 9159) and satellite-lock was lost. At this point, the navigation system mode changed to dead reckoning (DR) and the system relies on the INS for position measurement. Satellite lock was obtained a short distance later (frame 9495) but lost again (frame 9813). At frame 10632 the satellites were reacquired and carrier phase was established near the end of the run at frame 13139. The plot and mode changes in Figure 20 were generated automatically using the BMS analysis software.

Table 7 shows the elapsed time between navigation system mode changes. It took 5 seconds and 14 seconds to acquire satellites (time elapsed between DR and DGPS modes). The longer time reflects the additional time required to clear trees and regain satellite line-of-site. Once satellitelock was acquired, it took 44 seconds (time elapsed between DGPS and cpDGPS modes) to resolve the cycle integer ambiguity required for carrier phase operation. This agrees with the manufacturer's claim of less than a minute.

\begin{tabular}{|l|l|l|}
\hline elapsed $(\mathrm{sec})$ & \multicolumn{1}{|c|}{ new mode } & \multicolumn{1}{c|}{ frames } \\
\hline 0 & cpDGPS & 0 \\
\hline 163 & DR & 9159 \\
\hline 5 & DGPS & 9495 \\
\hline 5 & DR & 9813 \\
\hline 14 & DGPS & 10632 \\
\hline 44 & cpDGPS & 13139 \\
\hline
\end{tabular}

Table 7. Elapsed time between navigation system mode changes. 
Figure 21 is an enlarged view of the vehicle trajectory during section "a" in Figure 20. The trajectory appears distorted because the axes scales are different in order to maximize the range of the plot. The figure shows how the output of the Kalman filter tracks the INS, offset by the previous drift, when GPS lock is lost. The drift value remains constant while the GPS lock is lost. Each circle in the figure represents a GPS update.

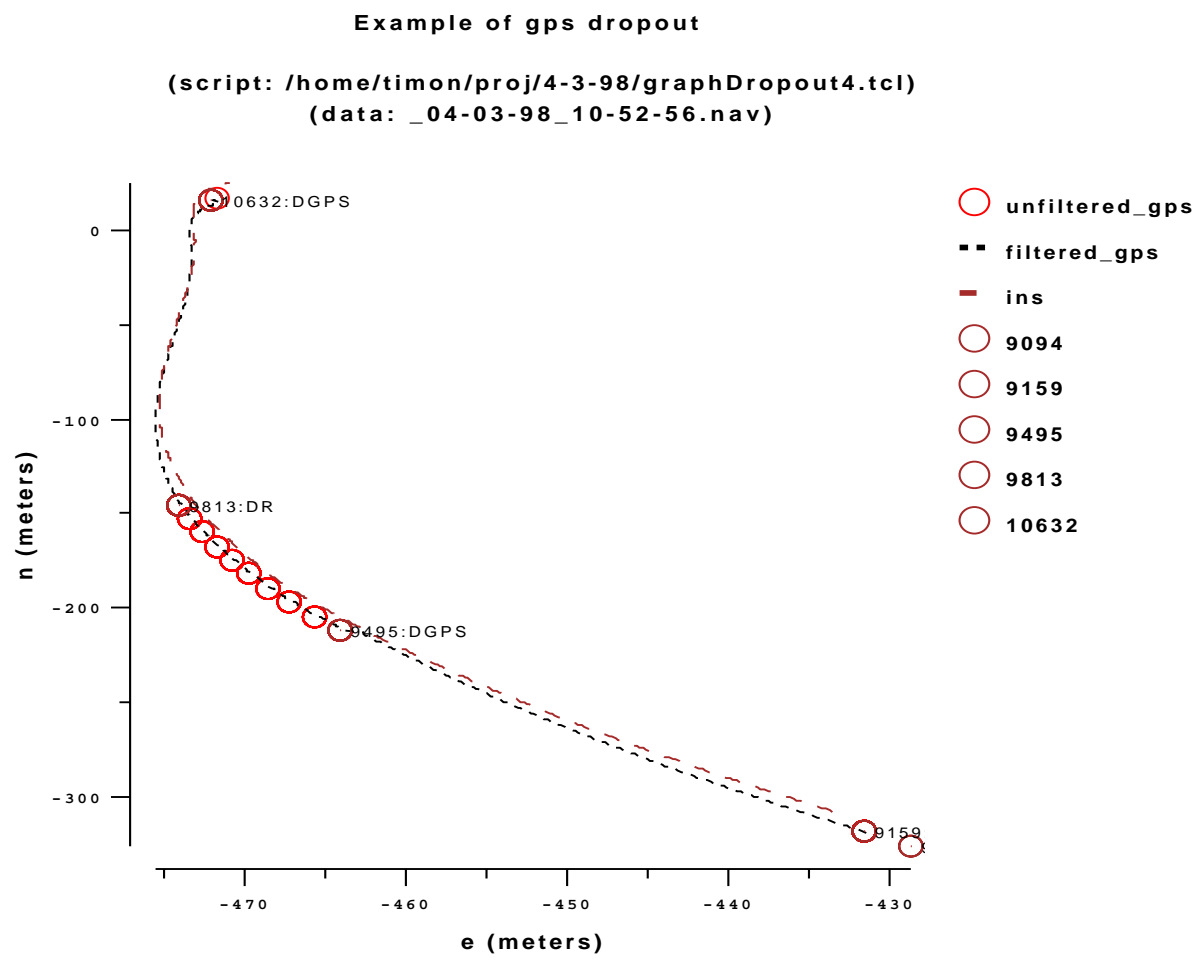

Figure 21. Vehicle position updates during section of trajectory labeled "a" in Figure 20. 
Figure 22 is an enlarged view of the vehicle trajectory during section "b" in Figure 20. The figure shows how the output of the Kalman filter converges to the GPS data once GPS satellite lock is obtained.

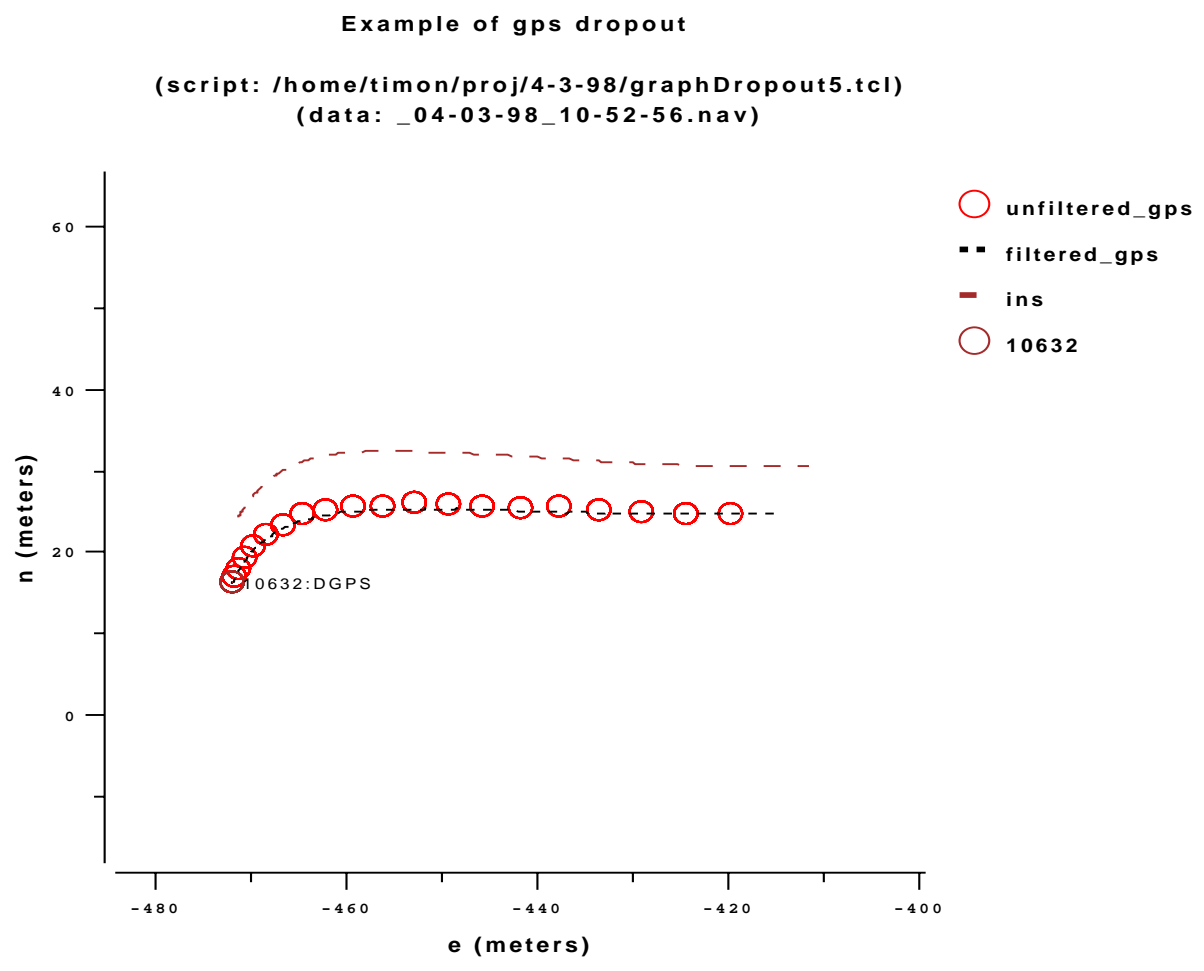

Figure 22. Vehicle position updates during section of trajectory labeled "b" in Figure 20. 
Figure 23 is an enlarged view of the vehicle trajectory during section "c" in Figure 20. The figure shows the position along the trajectory when the receiver achieved carrier phase lock. The Kalman filter converges even further when the vehicle slows to a stop.

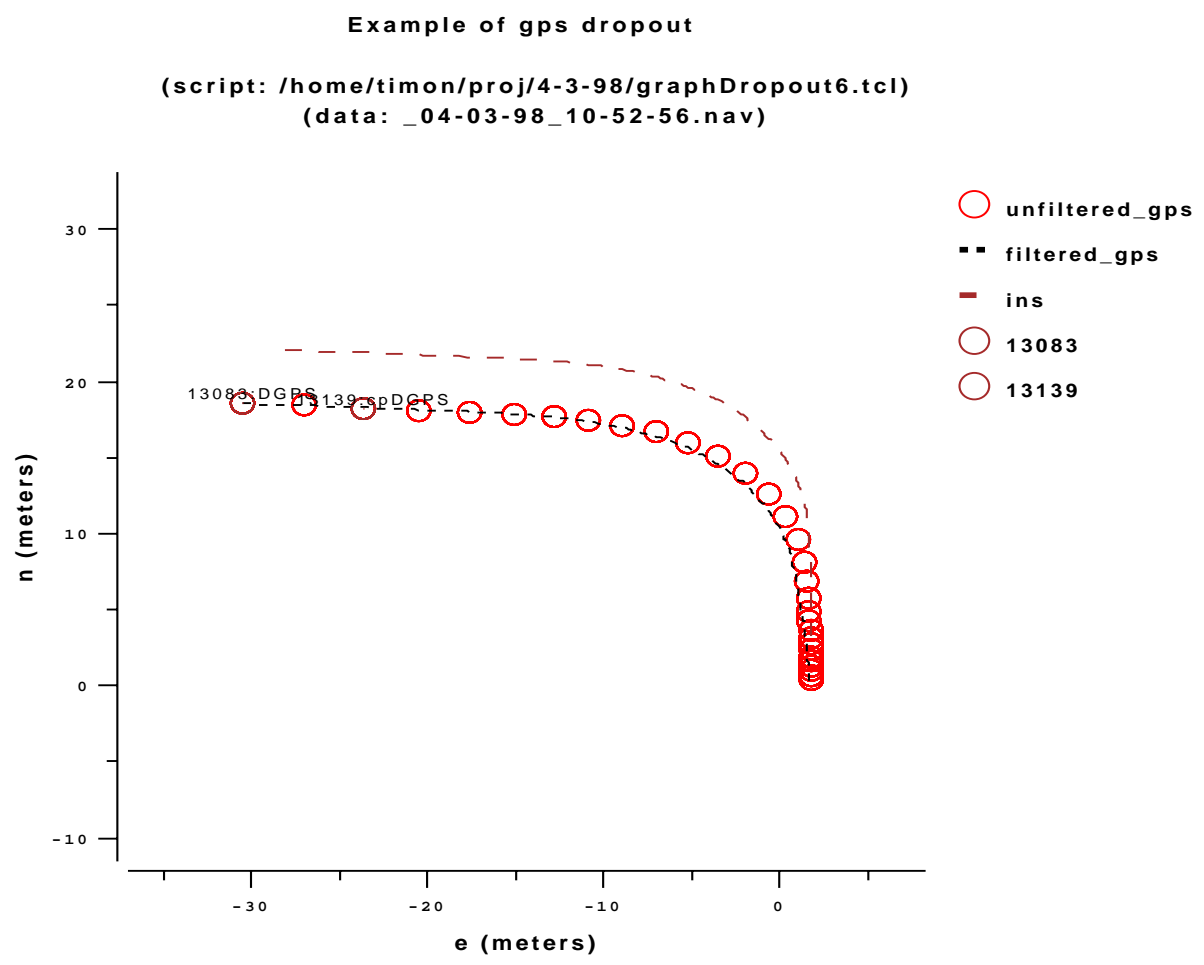

Figure 23. Vehicle position updates during section of trajectory labeled "c" in Figure 20. 
Finally, in Figure 24, the locations of the nail have been picked out from the video and transformed into GPS space. The origin of the graph is the manually surveyed location of the nail. The circles are the locations of the nail in the first and last (14456) video frame using the unfiltered (raw) GPS data. The pluses are the nails based on Kalman filtered output. Both sets of data are within 10 centimeters of the nail.

All Nails

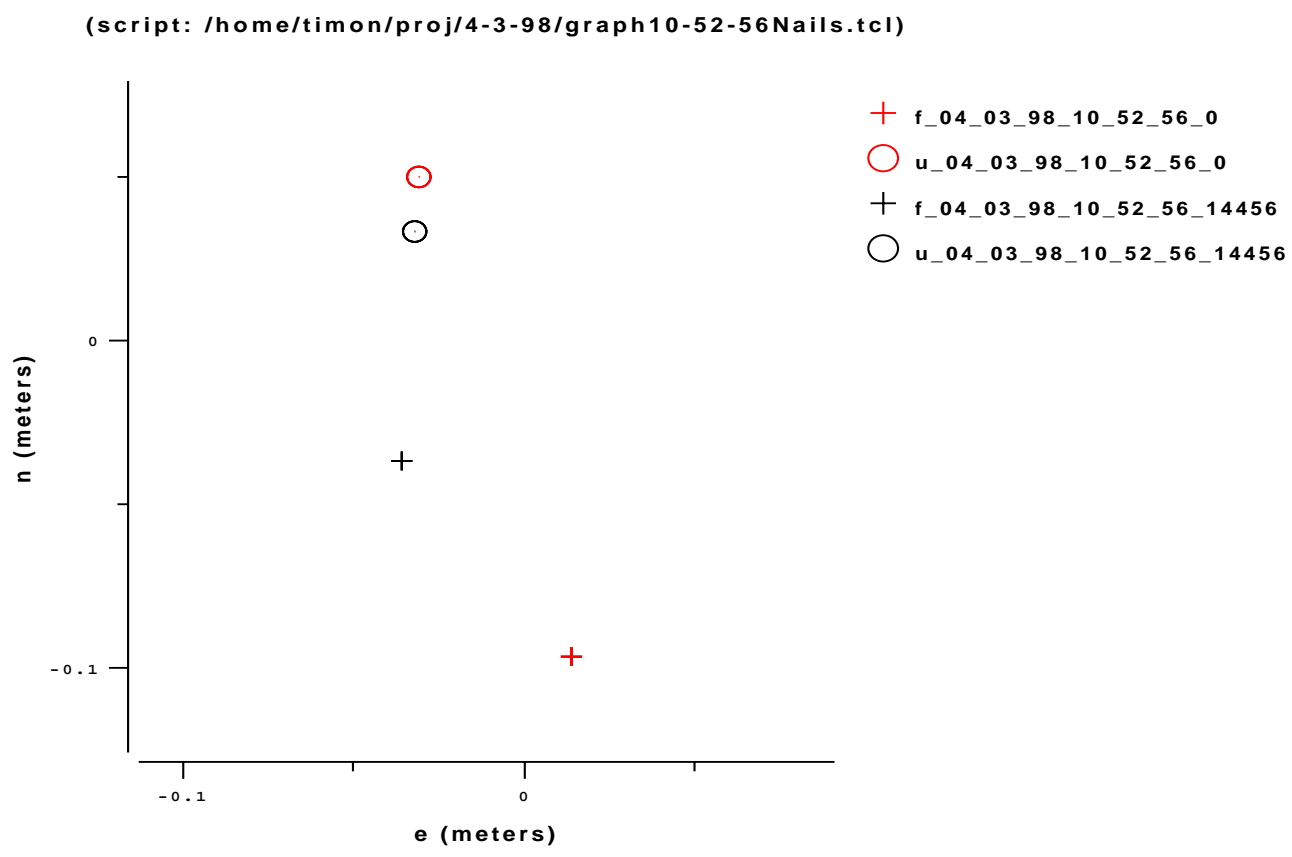

Figure 24. Nail locations, derived from navigation system (filtered_gps) and raw GPS (unfiltered_gps) data, at the beginning and end of run in Figure 20 


\subsection{Lane Position Measurements}

This test shows how the BMS can measure the location of the passenger-side lane marker while the vehicle is moving. A sequence of the recorded video data is processed and the lane location in each frame is extracted automatically using an adjustable template correlation function developed at Carnegie Mellon University ${ }^{6}$. The lane location is transformed into absolute GPS space. The lane and the vehicle locations are shown in Figure 25. The analysis software automatically labels the location of the minimum and maximum distances between the vehicle and the lane. The software can be extended to also flag when the vehicle crosses the lane.

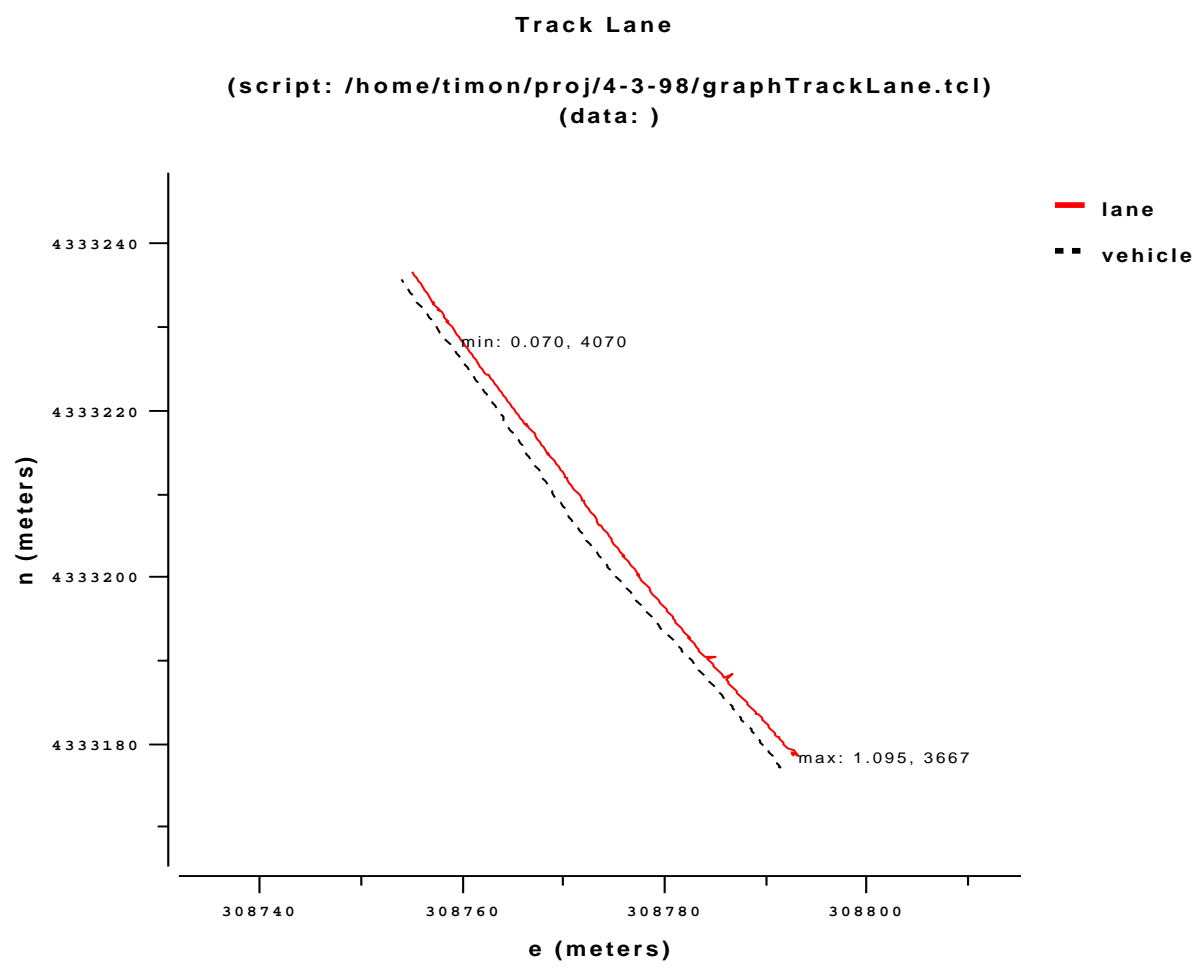

Figure 25. Trajectory of vehicle and location of outside lane marker shown with minimum and maximum distance between vehicle and lane labeled ( $\mathrm{min} / \mathrm{max}$ : distance from lane in meters, GPS record number). 


\section{Conclusion}

The thrust of the AUTONAV / DOT project has been to develop a quantitative means for measuring the performance of roadway departure warning systems. A series of performance measures describing the reliability of a warning system has been defined. A baseline measurement system (BMS) has been developed to gather, process and analyze data that is then used to compute the performance measures.

The BMS uses a carrier phase differential GPS receiver and an inertial navigation system (ring laser gyros and odometry) to measure vehicle position and a calibrated camera system to measure the vehicle's position relative to a lane marker. An interface is developed between the BMS and the warning system so that the output of the warning system can also be recorded. The output of the warning system, the camera and GPS is synchronized and stored digitally onto a large array of disks in real-time $(60 \mathrm{~Hz})$ during normal driving scenarios. Afterwards, the data is analyzed to determine when the vehicle departs a lane. The time of departure is compared against the output of the warning system. True positive and true negative outcomes indicate reliable operation, while false positive and false negative outcomes indicate unreliable operation. The tally of the outcomes is used to produce reliability, failure and false alarm rates, which are the primary performance metrics. 


\section{Appendix A: Autonav Program Description}

The goal of the U.S. Department of Defense, AUTONAV (Autonomous Vehicle Navigation Control System) project is to develop a control system and sensor suite that when mounted on a vehicle will enable the vehicle to autonomously navigate. The vehicles will be used to perform scouting and reconnaissance missions without putting the soldier in danger. The project team is integrating and evaluating several sensor and control system components that may be of use to the Department of Transportation. Below is a summary of the main components.

\section{UBM 4D Perception and Control System}

The UBM (Universität der Bundeswehr Munchen) 4D Perception and Control System ${ }^{7}$ is a system capable of a wide range of autonomous driving functions. The UBM system has demonstrated the ability to drive autonomously along a highway, to perceive traffic in front and to the sides, to maintain speed and spacing with a vehicle in front, and to pass vehicles when appropriate. A core component of the UBM system is the Road Detection and Tracking (RDT) module. The RDT is capable of looking forward, detecting lane markers, tracking the lane markers, and computing lane curvatures. The system is currently undergoing conversion to run on standard PC hardware and will be integrated on the NIST vehicle and evaluated in the 1999 time frame.

\section{DSRC Vision Front End}

The David Sarnoff Research Center VFE ${ }^{8}$ is a special-purpose vision processor that can perform complex operations on video in real-time. Two of the primary functions of the VFE for AUTONAV is image stabilization and stereo processing. Image stabilization is done electronically by detecting global motion in the image and then warping the image to subtract out the motion. Stabilization is an important preprocessing step to any countermeasure system based on video. Stabilization helps simplify image processing algorithms and improves accuracy. Stereo processing takes images from two cameras and produces a single range image. Each pixel in the image is the range along the two cameras' view axis. Range images form the basis for collision detection and countermeasure systems. The system will be integrated on the vehicle and tested in the 1999 time frame.

\section{EBK LADAR}

EBK $^{9}$ (Entfernungs-Bild-Kamera) LADAR, manufactured by Dornier, uses a laser and a two degree-of-freedom scanning mechanism to collect range measurements. The ladar produces $128 \times 64$ (horizontal x vertical pixels) range image once each second, though each pixel is available immediately upon measurement. The maximum range is $50 \mathrm{~m}$ and the range resolution is $6 \mathrm{~cm}$. The ladar produces range images similar to a stereo system but with different characteristics. The primary drawback is the time it takes to scan the laser mechanically and to make the measurements. The advantage of ladar over stereo is the ability to produce more robust readings under a wider variety of conditions. Tests are now being conducted in the AUTONAV project to determine the performance of stereo systems versus ladar. NIST is also looking at flash ladar systems that do not require a mechanically scanned laser. A flash ladar illuminates 
the entire scene and measures range simultaneously throughout the image. Typical flash ladars currently under development have the ability to produce up to a 320x240 range image at $10 \mathrm{~Hz}$. The range images from a ladar form the basis for collision detection and collision avoidance countermeasure systems. 


\section{$\underline{\text { References }}$}

${ }^{1}$ K. Murphy, et al., "Ground Vehicle Control at NIST: from Teleoperation to Autonomy”, Seventh Annual Space Operations, Applications, and Research Symposium, Houston, TX, August 3-5, 1993.

${ }^{2}$ H-726 Modular Azimuth Position System Technical Description, December 1992, Honeywell Avionics Division, St. Petersburg, FL.

${ }^{3}$ K. Konstatinides and J. Rasure, "The Khoros Software Development Environment", Journal of Visual Languages and Computing, pp. 217-246, 1991.

${ }^{4}$ B. N. Taylor and C. E. Kuyatt, "Guidelines for Evaluating and Expressing the Uncertainty of NIST Measurement Results", NIST Technical Note 1297, 1994 Edition. http://physics.nist.gov/cuu/Uncertainty/index.html.

${ }^{5}$ Ashtech Supplement to Z-12 Receiver Operating Manual Covering RTZ Functions, March 1995. Phone 1-800-2292400.

${ }^{6}$ M. Chen, D. Pomerleau, T. Jochem, “Aurora: A Vision-Based Roadway Departure Warning System ”, Proceedings of IEEE International Conference on Intelligent Robots and Systems, Pittsburgh, PA, August 1995. Also appears in "Run-Off-Road Collision Avoidance Using IVHS Countermeasures, Task 3, Volume 1", Contract No. DTNH22-93C-07023, USDOT National Highway Traffic Safety Administration, Office of Collision Avoidance Research, August 1995, pg. 14.

${ }^{7}$ E.D. Dickmanns, et al.,"The Seeing Passenger Car 'VaMoRs-P'", International Symposium on Intelligent Vehicles '94, October 24-26, 1994, Paris.

${ }^{8}$ P.J. Burt, P. Anandan and K.J. Hanna, "An Electronic Front End Processor for Active Vision", SPIE Conference on Intelligent Robotics, November 1992.

${ }^{9}$ Dornier GmbH, Box 1420, 7990 Friedrichshafen 1, Germany. Phone: 07545-89969. 